517.938

doi:10.13108/2018-10-1-25

UDC 517.938

\title{
OPERATOR METHODS FOR CALCULATING LYAPUNOV VALUES IN PROBLEMS ON LOCAL BIFURCATIONS OF DYNAMICAL SYSTEMS
}

\author{
N.I. GUSAROVA, S.A. MURTAZINA, M.F. FAZLYTDINOV, M.G. YUMAGULOV
}

\begin{abstract}
In the work we consider basic scenarios of local bifurcations in dynamical systems. We study the systems described by autonomous differential equations, discrete equations, as well as by non-autonomous periodic equations. We provide new formulae for calculating Lyapunov values. The formulae are obtained on the basis of a general operator approach for studying local bifurcations and they do not assume passing to normal forms and using the theorems on a central manifold. This method allows us to obtain new bifurcation formulae for studying main scenarios of local bifurcations. In the work we show how these bifurcation formulae lead one to new formulae for calculating Lyapunov values in problems on equilibria bifurcation, in Andronov-Hopf problems, in problems of doubling period, in problems on forced oscillations, etc.

In the paper, the main attention is paid to obtain the first and the second Lyapunov value. The proposed approach allows us obtain Lyapunov values of higher order. As an application of the obtained formulae, in the paper we analyze basic scenarios of local bifurcations. We consider the problems on the direction of bifurcations, on stability of emerging solutions, on leading asymptotics for the solutions, etc. As an example, we calculate the Lyapunov values for Andronov-Hopf bifurcation in Langford system and for the problems on doubling period in Henon model.
\end{abstract}

Keywords: dynamical systems, bifurcation, Lyapunov values, equilibrium, stability.

Mathematics Subject Classification: 37G10, 37G15

\section{INTRODUCTION}

A key role in the bifurcation theory of dynamical systems is played by so-called Lyapunov values allowing one to determine important properties of the bifurcations like the stability of emerging solutions, the direction of bifurcations, etc. The calculating of Lyapunov values is important also from the point of view of applications, for instance, in studying the behavior of dynamical system for the parameters close to the boundary of the stability domain (safe and unsafe boundaries).

There is a series of approaches allowing one to calculate the Lyapunov values. Here we mention the following ones. The first approach is classical and usually exactly this approach is employed for formal calculating of Lyapunov values. This approach relates to using the theorem of central manifold and the normal form method (see [1]-[7]). In studying the main scenarios of local bifurcations, this approach allows one to reduce the original equations to a rather simple (canonical) form and the nonlinearity coefficients in this form determine the Lyapunov values. The obtained formulae turn out to be very effective for analysing the bifurcation, which

N.I. Gusarova, S.A. Murtazina, M.F. Fazlytdinov, M.G. Yumagulov, Operator methods for CALCULATING LYAPUNOV QUANTITIES IN PROBLEMS ON LOCAL BIFURCATIONS OF DYNAMICAL SYSTEMS.

(c) Gusarova N.I., Murtazina S.A., Fazlytdinov M.F., Yumagulov M.G. 2018.

Submitted March 6, $201 \%$. 
was demonstrated in a series of works. Here we specially mention works [1], [2] and [7], in which the detailed study of main bifurcation scenarios was done depending on the Lyapunov values. However, we should say that employing such formulae for studying particular equations requires, as a rule, the preliminary transformation of given equation and quite often this is a non-trivial problem.

Another approach is aimed on calculating Lyapunov values in terms of given equations. It is often used in applications. The works by many authors were devoted to obtaining the formulae and algorithms for calculating the Lyapunov values, see, for instance, [1, [2] and the references therein. Although here the obtained formulae are quite complicated, their main advantage is the fact that they allow one to analyse the bifurcations in terms of the given equations.

We should also mention the approaches based on using modern computer techniques and the programs for symbolic calculations. These approaches made an essential progress in studying Lyapunov values, in particular, in calculating the values of third and higher orders. While the explicit expressions for the first and second Lyapunov values for many bifurcation scenarios were obtained in 1940-1950, the expression for the next Lyapunov values in symbolic forms were obtained rather recently (see, for instance, [8], [9] and the references therein).

The question which of the approaches is better has no definite answer since various classes of problem possess various properties and therefore, in some situation one method is preferable, while another is better in another situation. One should also remember that the applied methods give the same final formula provided they are properly compared.

The results of the present work relate to the second approach. We propose a general scheme allowing us to obtain new formulae for Lyapunov values in problems on main scenarios of local bifurcations of dynamical systems in terms of the given equations. The provided formulae allows us not only to calculate effectively the Lyapunov values, but also to make a new study of the bifurcation properties.

The proposed formulae for calculating Lyapunov values are obtained on the base of the general operator approach for studying local bifurcations of dynamical systems; the main aspects of this method were exposed in [10]-[14]. In particular, this method allows us obtain new bifurcations formulae for the main bifurcation scenarios. In their turn, these formulae allows to study effectively the bifurcations and to answer the most important questions on the bifurcations properties: transversality condition, direction of bifurcation, stability of emerging solutions, leading terms in the asymptotics for solutions, etc. These bifurcation formulae turned out to be closely related with the formulae for Lyapunov values that is shown in the present work.

The paper is organised as follows. In Sections 2-4 we consider dynamical systems described by autonomous differential equations (Section 2), by discrete equations (Section 3) and nonautonomous periodic equations (Section 4). Here we provide new formulae for Lyapunov values, discuss some properties of the bifurcations and the obtained results are demonstrated by examples. In Section 5 we provide the proof of the main results of the work.

\section{Autonomous differential Equations}

In this section we calculate Lyapunov values for dynamical systems described by the autonomous differential equation

$$
x^{\prime}=F(x, \mu), \quad x \in \mathbb{R}^{N},
$$

in which $\mu$ is a scalar parameter, $F(x, \mu)$ is a continuously differentiable in $x$ and $\mu$ function.

2.1. Bifurcation and central manifold. Assume that for some $\mu=\mu_{0}$ equation (1) has the equilibrium $x=0$, that is, $F\left(0, \mu_{0}\right)=0$. Then equation (1) can be represented as

$$
x^{\prime}=A(\mu) x+b(x, \mu)+u(\mu), \quad x \in \mathbb{R}^{N},
$$


where $A(\mu)=F_{x}^{\prime}(0, \mu)$ is the Jacobi matrix, $u(\mu)=F(0, \mu)$, the function $b(x, \mu)$ obeys the condition $\|b(x, \mu)\|=o(\|x\|)$ as $x \rightarrow 0$ uniformly in $\mu$, and the function $u(\mu)$ obeys the condition $u\left(\mu_{0}\right)=0$.

In what follows we suppose that the function $b(x, \mu)$ is of the form:

$$
b(x, \mu)=b_{2}(x, \mu)+b_{3}(x, \mu)+b_{4}(x, \mu),
$$

where $b_{2}(x, \mu)$ involves quadratic in $x$ terms, $b_{3}(x, \mu)$ involves cubic terms and $b_{4}(x, \mu)$ is smooth and satisfies the condition $\left\|b_{4}(x, \mu)\right\|=O\left(\|x\|^{4}\right)$ as $x \rightarrow 0$ uniformly in $\mu$.

If the Jacobi matrix $A_{0}=A\left(\mu_{0}\right)$ has one or several eigenvalues with zero real parts, then $\mu_{0}$ is a bifurcation point of system (1). In this case, as the parameter $\mu$ passes through $\mu_{0}$, the phase portrait of system (1) in the vicinity of the point $x=0$ usually transforms qualitatively. A huge amount of works was devoted to studying various bifurcation scenarios, see, for instance, [1], 2], 7]-[10]. In these works, there was proposed a series of effective methods like the normal forms method, the methods based on the central manifolds theory, the method of parameter functionalization, etc.

According the theorem on central manifold, see, for instance, [1], 2], the problem on local bifurcations for $N$-dimensional system (1) can be reduced to an equivalent (in the natural formulation) problem for a system of a lower dimension. In view of this, we mention some notions and facts which will be used later.

Assume that the spectrum $\sigma$ of the matrix $A_{0}$ consists of two non-empty parts: $\sigma=\sigma_{0} \cup \sigma^{0}$, where $\sigma_{0}$ contains eigenvalues with zero real parts, and $\sigma^{0}$ are other eigenvalues. By $E_{0}$ and $E^{0}$ we denote the roots subspaces of the matrix $A_{0}$ associated respectively with the parts $\sigma_{0}$ and $\sigma^{0}$ of its spectrum. Let $k_{0}$ and $k^{0}$ be the dimensions of the subspaces $E_{0}$ and $E^{0}$; then $k_{0}+k^{0}=N$ and $1 \leqslant k_{0}, k^{0} \leqslant N-1$. The space $\mathbb{R}^{N}$ can be represented as the direct sum $\mathbb{R}^{N}=E_{0} \bigoplus E^{0}$ of the subspaces $E_{0}$ and $E^{0}$ invariant for the operator $A_{0}: \mathbb{R}^{N} \rightarrow \mathbb{R}^{N}$. Finally, we denote by $P_{0}: \mathbb{R}^{N} \rightarrow E_{0}$ and $P^{0}: \mathbb{R}^{N} \rightarrow E^{0}$ the corresponding projectors.

According the theorem on central manifold, there exists a $\delta_{1}$-neighbourhood $T\left(0, \delta_{1}\right)$ of the point $x=0$ and a $\delta_{2}$-neighbourhood of the number $\mu_{0}$ such that as $\left|\mu-\mu_{0}\right|<\delta_{2}$, system (1) possesses a smooth invariant $k_{0}$-dimensional manifold $W(\mu)$ in $T\left(0, \delta_{1}\right)$ containing the point $x=0$ and touching the subspace $E_{0}\left(\right.$ as $\left.\mu=\mu_{0}\right)$ at the point $x=0$. The invariance of the manifold $W(\mu)$ for system (1) means that if at some time, some its motion is located on the manifold $W(\mu)$, it will stay on the manifold $W(\mu)$ at all other times until this motion stays in the ball $T\left(0, \delta_{1}\right)$. The manifold $W(\mu)$ is called central; it can be defined by the equation of form $v=\psi(u, \mu)$, where $u \in E_{0}, v \in E^{0}$, and the function $\psi(u, \mu)$ is smooth and satisfies the identities $\psi\left(0, \mu_{0}\right)=0, \psi_{u}^{\prime}\left(0, \mu_{0}\right)=0$.

By projecting into the subspaces $E_{0}$ and $E^{0}$, in the vicinity of the point $x=0$, equation (1) can be represented as the equivalent system

$$
\left\{\begin{array}{l}
u^{\prime}=f(u, v, \mu) \\
v^{\prime}=g(u, v, \mu)
\end{array}\right.
$$

where $u=P_{0} x, v=P^{0} x$, and $f$ and $g$ are smooth functions taking values in $E_{0}$ and $E^{0}$, respectively. These functions can be represented as

$$
f(u, v, \mu)=A_{0} u+\xi(u, v, \mu), \quad g(u, v, \mu)=A_{0} v+\eta(u, v, \mu),
$$

where the functions $\xi(u, v, \mu)$ and $\eta(u, v, \mu)$ satisfy the relations:

$$
\left\{\begin{array}{lll}
\xi\left(0,0, \mu_{0}\right)=0, & \eta\left(0,0, \mu_{0}\right)=0, & \xi_{u}^{\prime}\left(0,0, \mu_{0}\right)=0 \\
\xi_{v}^{\prime}\left(0,0, \mu_{0}\right)=0, & \eta_{u}^{\prime}\left(0,0, \mu_{0}\right)=0, & \eta_{v}^{\prime}\left(0,0, \mu_{0}\right)=0 .
\end{array}\right.
$$


Thus, the problem on local bifurcations in $N$-dimensional equation (1) can be reduced to studying $k_{0}$-dimensional equation:

$$
u^{\prime}=G(u, \mu), \quad u \in E_{0},
$$

where $G(u, \mu)=f(u, \psi(u, \mu), \mu)$. This equation involves all principal features of the bifurcation scenario in initial equation (1). In particular, the analysis of equation (6) (usually by means of the normal forms method) leads one to the notion of the Lyapunov values. In what follows, we discuss this issue while considering main bifurcation scenarios.

In the present paper the problem on Lyapunov values is studied in the following main cases:

S1. The matrix $A_{0}$ has a simple eigenvalue 0 ;

S2. The matrix $A_{0}$ has a pair of simple eigenvalues of form $\pm \omega_{0} i$, where $\omega_{0}>0$.

We assume that other eigenvalues of the matrix $A_{0}$ has non-zero real parts.

We note that in studying local bifurcations in S2 as well as in some subcases in S1 one usually assumes that the function $u(\mu)$ in equation (2) is zero, that is, this equation reads as

$$
x^{\prime}=A(\mu) x+b(x, \mu), \quad x \in \mathbb{R}^{N},
$$

where $b(x, \mu)$ is determined by identity (3).

2.2. Case S1: bifurcations of equilibria. We consider first case S1. In this case the qualitative transformation of the behavior of system (1) in the vicinity of the point $x=0$ as the parameter $\mu$ passes through $\mu_{0}$ consists usually in emerging of non-zero equilibria. Such transformation will be called equilibria bifurcation of system (1).

In case S1, equation (6) is one-dimensional and by assumption (3), for $\mu=\mu_{0}$ the function $G(u, \mu)$ can be represented as

$$
G\left(u, \mu_{0}\right)=l_{1} u^{2}+l_{2} u^{3}+o\left(u^{3}\right),
$$

see, for instance, [1]. In other words, equation (6) for $\mu=\mu_{0}$ is of the form

$$
u^{\prime}=l_{1} u^{2}+l_{2} u^{3}+o\left(u^{3}\right) .
$$

The numbers $l_{1}$ and $l_{2}$ are called respectively first and second Lyapunov value in the problem on equilibria bifurcation in system (1).

Remark 1. Generally speaking, the first and second Lyapunov values $l_{1}$ and $l_{2}$ are determined non-uniquely. This is due to the fact that the basis in subspaces $E_{0}$ and $E^{0}$ (see the previous subsection) can be chosen in various ways and hence, the function $G\left(u, \mu_{0}\right)$ can be different, but in all cases of form (8). However, we can show that if for some choice of the basis the number $l_{1}$ (or $l_{2}$ ) is non-zero, it is non-zero in each other case. At that, the sign of the number $l_{1}$ can change, while the sign of the number $l_{2}$ is kept.

Let us provide a statement allowing one to calculate the Lyapunov values $l_{1}$ and $l_{2}$ in terms of initial equation (1). We denote by $e$ and $g$ the eigenvectors of the matrix $A_{0}$ and the transposition of the matrix $A_{0}^{*}$, respectively, associated with the eigenvalue 0 . These vectors can be chosen according the identities

$$
\|e\|=1, \quad(e, g)=1 .
$$

Theorem 1. Assume that the matrix $A_{0}$ has the simple eigenvalue 0 and its other eigenvalues are not located at the imaginary axis. Then the first Lyapunov value of system (1) in the problem of bifurcation of equilibrium is equal to $l_{1}=\left(b_{2}\left(e, \mu_{0}\right), g\right)$. If $b_{2}(x, \mu) \equiv 0$, then $l_{1}=0$ and $l_{2}=\left(b_{3}\left(e, \mu_{0}\right), g\right)$.

The proof of this and other main statements is given in Section 5 . 
Remark 2. Theorem 1 concerns calculating only the first and second Lyapunov values $l_{1}$ and $l_{2}$. This is due to assumption (3), according to which the nonlinearity $b(x, \mu)$ involves only the terms $b_{2}(x, \mu)$ and $b_{3}(x, \mu)$ of second and third power. If the terms of higher order are assumed to be given, we can obtain similar statements for the next Lyapunov values and, in particular, we can consider the situations when several first Lyapunov values vanish simultaneously.

Remark 3. It is obvious that there are just two options for choosing the normalization for the vectors $e$ and $g$ in accordance with identities (9): these options differ by the sign only. This is why in Theorem 1 we provide in fact two versions of the formulae for Lyapunov values $l_{1}$ and $l_{2}$. Apart of the version provided in the theorem, these the following formulae: $l_{1}=$ $\left(b_{2}\left(-e, \mu_{0}\right),-g\right)=-\left(b_{2}\left(e, \mu_{0}\right), g\right)$ and $l_{2}=\left(b_{3}\left(-e, \mu_{0}\right),-g\right)=\left(b_{3}\left(e, \mu_{0}\right), g\right)$. In other words, in the mentioned two cases the numbers $l_{1}$ differ only by the sign, while the numbers $l_{2}$ coincide.

The equilibria bifurcation of system (1) can follow various scenarios. The main of them are saddle-node bifurcation, transcritical bifurcation and pitchfork bifurcation. Let us provide some properties of the mentioned bifurcation scenarios; the proof of these properties employ Theorem 1 .

2.2.1. Saddle-node bifurcation. The model example of the saddle-node bifurcation is given by the scalar equation $x^{\prime}=\mu-x^{2}$. As $\mu<0$, this equation has no equilibria, as $\mu=0$ it has only the zero equilibrium, while as $\mu>0$, it has two non-zero equilibria $x= \pm \sqrt{\mu}$. Thus, as $\mu$ passes the value $\mu=0$, in the vicinity of the point $x=0$ there emerges first $($ as $\mu=0)$ the single equilibrium $x=0$ for this equation and then (as $\mu>0$ ), this equilibrium "splits" into two non-zero equilibria $x_{1,2}= \pm \sqrt{\mu}$; one of them is stable, while the other is unstable. We can assume here that the first Lyapunov value is equal to $l_{1}=-1$.

A similar scenario is the saddle-node bifurcation in equation (1) for arbitrary $N \geqslant 1$ in the case, when the first Lyapunov value $l_{1}$ is non-zero. This relates to fusion (and then disappearing) of two equilibria, one of which is of type "node", while the other is of type "saddle". Let us provide the corresponding statement implied by the results of work [10].

Theorem 2. Suppose that under the assumptions of Theorem 1 the relations

$$
l_{1}=\left(b_{2}\left(e, \mu_{0}\right), g\right) \neq 0, \quad u_{1}=\left(u^{\prime}\left(\mu_{0}\right), g\right) \neq 0
$$

hold. Let $\mu_{1} \equiv-l_{1} / u_{1}>0$. Then there exists $\delta>0$ such that

1. As $\mu \in\left(\mu_{0}-\delta, \mu_{0}\right)$, equation (1) has no equilibria in the $\delta$-neighbourhood of the point $x=0$, while for each $\mu \in\left(\mu_{0}, \mu_{0}+\delta\right)$ it has two non-zero equilibria $x=x_{1}(\mu)$ and $x=x_{2}(\mu)$.

2. The functions $x=x_{1}(\mu)$ and $x=x_{2}(\mu)\left(x_{1}\left(\mu_{0}\right)=x_{2}\left(\mu_{0}\right)=0\right)$ defined for $\mu \in\left[\mu_{0}, \mu_{0}+\delta\right)$ are continuously differentiable and as $\mu=\mu_{0}$, and the eigenvector $e$ of the matrix $A_{0}$ associated with the eigenvalue 0 is tangent for thecir graphs.

3. Let the non-zero eigenvalues of the matrix $A_{0}$ have negative real parts, then one of the graphs of the functions $x=x_{1}(\mu)$ and $x=x_{2}(\mu)$ contains the asymptotically stable equilibria, while the other does the unstable ones.

A similar statement can be given also for the case $\mu_{1}<0$. In this case only the bifurcation direction changes, that is, the non-zero equilibria $x=x_{1}(\mu)$ and $x=x_{2}(\mu)$ emerge as $\mu \in$ $\left(\mu_{0}-\delta, \mu_{0}\right)$.

Thus, if the first Lyapunov value $l_{1}$ is non-zero, then as rule, in system (1) the usual scenario of saddle-node bifurcation is realized, in which, in the vicinity of the point $x=0$ there exists no equilibria of system (1) as $\mu<\mu_{0}$ (or as $\mu>\mu_{0}$ ) and there exist two equilibria for each $\mu>\mu_{0}$ (or as $\left.\mu<\mu_{0}\right)$.

We note that if $l_{1}=0$ and $l_{2} \neq 0$, then as a rule, in system (1) the scenario of saddle-node bifurcation is realized, in which in the vicinity of the point $x=0$ there exists a single non-zero equilibrium of system (1) both as $\mu<\mu_{0}$ and as $\mu>\mu_{0}$. 
2.2.2. Transcritical bifurcation and pitchfork bifuraction. In the framework of case S1, we assume that we have $u(\mu) \equiv 0$ in equation (2). In other words, we consider equation (7). For all $\mu$, the point $x=0$ is the equilibrium for this equation. Then the main bifurcation scenarios of system (7) in the vicinity of the point $x=0$ are transcritical bifurcaiton and pitchfork bifurcation.

The model example of the transcritical bifurcation is given by the scalar equation $x^{\prime}=\mu x-x^{2}$. For all $\mu$, it has equilibrium $x=0$. As $\mu$ passes through $\mu=0$, there emerges the non-zero equilibrium $x=\mu$ for this equation in the vicinity of the point $x=0$. This equilibrium is stable for $\mu>0$ and unstable for $\mu<0$. Here the first Lyapunov value is equal to $l_{1}=-1$.

A model example for the pitchfork bifurcation is provided by the scalar equation $x^{\prime}=\mu x-x^{3}$. This equation also has the equilibrium $x=0$ for all $\mu$. For $\mu<0$ the system has no other equilibrium, while as $\mu$ passes through $\mu=0$, in the vicinity of the point $x=0$ there arise two non-zero equilibria $x= \pm \sqrt{\mu}$ of this equation and these equilibria are stable. Here the Lyapunov values are $l_{1}=0$ and $l_{2}=-1$.

For arbitrary $N \geqslant 1$, the scenarios of transcritical bifurcation and pitchfork bifurcation in equation (1) are similar. At that, if $l_{1} \neq 0$, the transcritical bifurcation occurs, while if $l_{1}=0$ and $l_{2} \neq 0$, the pitchfork bifurcation arises.

Let us provide some properties of transcritical bifurcation implied by the results of work [10].

Theorem 3. Suppose that under the assumptions of Theorem 1 the relations

$$
l_{1}=\left(b_{2}\left(e, \mu_{0}\right), g\right) \neq 0, \quad \gamma_{1}=\left(A^{\prime}\left(\mu_{0}\right) e, g\right) \neq 0
$$

hold true. Then there exists $\delta>0$ such that

1. For each $\mu \in\left(\mu_{0}, \mu_{0}+\delta\right)$ and $\mu \in\left(\mu_{0}-\delta, \mu_{0}\right)$, equation (7) has exactly one non-zero equilibrium $x=x(\mu)$ in the $\delta$-neighbourhood of the point $x=0$.

2. The function $x=x(\mu)\left(x\left(\mu_{0}\right)=0\right)$ defined for $\mu \in\left(\mu_{0}-\delta, \mu_{0}+\delta\right)$ is continuously differentiable and as $\mu=\mu_{0}$, the eigenvector $e$ of the matrix $A_{0}$ associated with the eigenvalue 0 is tangent to the graph of this function.

3. Let $\gamma_{1}<0\left(\gamma_{1}>0\right)$ and the non-zero eigenvalues of the matrix $A_{0}$ has negative real parts; then the graph of the function $x=x(\mu)$ contains unstable (asymptotically stable) equilibria as $\mu>\mu_{0}$ and asymptotically stable (unstable) equilibria as $\mu<\mu_{0}$.

In particular, this theorem implies that the qualitative properties of the transcritical bifurcation are independent of the sign of the first Lyapunov value $l_{1}$, which is natural in view of Remarks 1 and 3 .

Let us provide some properties of the pitchfork bifurcation implied by the results of work [10].

Theorem 4. Suppose that under the assumptions of Theorem 1 the relations

$$
l_{1}=\left(b_{2}\left(e, \mu_{0}\right), g\right)=0, \quad l_{2}=\left(b_{3}\left(e, \mu_{0}\right), g\right) \neq 0, \quad \gamma_{1}=\left(A^{\prime}\left(\mu_{0}\right) e, g\right) \neq 0
$$

hold. Let $\mu_{2} \equiv-l_{2} / \gamma_{1}>0$. Then there exists $\delta>0$ such that

1. For each $\mu \in\left(\mu_{0}, \mu_{0}+\delta\right)$, equation (7) has exactly two non-zero equilibria $x=x_{1}(\mu)$, $x=x_{2}(\mu)$ in the $\delta$-neighbourhood of the point $x=0$, while for each $\mu \in\left(\mu_{0}-\delta, \mu_{0}\right)$ this equation has no non-zero equilibria.

2. The functions $x=x_{1}(\mu)$ and $x=x_{2}(\mu)\left(x_{1}\left(\mu_{0}\right)=x_{2}\left(\mu_{0}\right)=0\right)$ defined as $\mu \in\left[\mu_{0}, \mu_{0}+\delta\right)$ are continuously differentiable and as $\mu=\mu_{0}$, the eigenvector e of the matrix $A_{0}$ associated with the eigenvalue 0 is tangent to the graphs of these functions.

3. Let $l_{2}<0\left(l_{2}>0\right)$ and let the non-zero eigenvalues of the matrix $A_{0}$ have negative real parts; then for each $\mu \in\left(\mu_{0}, \mu_{0}+\delta\right)$ the equilibria $x=x_{1}(\mu)$ and $x=x_{2}(\mu)$ of equation (7) are asymptotically stable (unstable). 
A similar statement can be provided for the case $\mu_{2}<0$. In this case only the bifurcation direction changes but not the stability property, that is the non-zero equilibria $x=x_{1}(\mu)$ and $x=x_{2}(\mu)$ emerge as $\mu \in\left(\mu_{0}-\delta, \mu_{0}\right)$, and if $l_{2}<0\left(l_{2}>0\right)$, these solutions are asymptotically stable (unstable).

2.3. Case S2: Andronov-Hopf bifurcation. We continue studying equation (7). Assume that Case $\mathrm{S} 2$ holds, that is, the matrix $A_{0}$ has a pair of simple pure imaginary eigenvalues $\pm \omega_{0} i$ $\left(\omega_{0}>0\right)$ and it has no other eigenvalue on the imaginary axis. In this case the main bifurcation scenario is the Andronov-Hopf bifurcation, which is the emergence of non-stationary periodic solutions $x(t, \mu)$ of small amplitude to equation (7) as the parameter $\mu$ passes through $\mu_{0}$. The Andronov-Hopf bifurcation is possible only as $N \geqslant 2$.

As a rule, for small $\left|\mu-\mu_{0}\right|$, the bifurcating solutions $x(t, \mu)$ of equation (7) emerge in one of three cases: (S1) $\mu>\mu_{0}$; (S2) $\mu<\mu_{0}$; (S3) $\mu=\mu_{0}$. The latter case is called degenerate; it is typical for linear and conservative systems. The former two cases hold under some nondegeneracy condition or non-linear term (3) in the right hand side in equation (7); one of the versions of such condition will be given below. Under this condition, in Cases (S1) and (S2), to each $\mu$, exactly one non-zero cycle $x(t, \mu)$ of a small amplitude is associated. At that, the function $x(t, \mu)$ depends smoothly on $\mu$ and the relation holds: $\max _{t}\|x(t, \mu)\| \rightarrow 0$ as $\mu \rightarrow \mu_{0}$. Finally, the period $T(\mu)$ of the solutions $x(t, \mu)$ depends smoothly on $\mu$ and the relation holds: $T(\mu) \rightarrow T_{0}$ as $\mu \rightarrow \mu_{0}$. Here $T_{0}=2 \pi / \omega_{0}$.

In the problem on the Andronov-Hopf bifurcation, equation (6) is two-dimensional. As $\mu=\mu_{0}$, this equation can be represented as

$$
\left\{\begin{array}{l}
u_{1}^{\prime}=-\omega_{0} u_{2}+f_{1}\left(u_{1}, u_{2}\right), \\
u_{2}^{\prime}=\omega_{0} u_{1}+f_{2}\left(u_{1}, u_{2}\right),
\end{array}\right.
$$

where the functions $f_{1}$ and $f_{2}$ vanish at the point $u=0$ together with its first derivatives and these functions satisfy representations similar to (3).

According the normal forms theory, see, for instance, [1, 2, 6], there exists a polynomial change of variables close to the identical one in the vicinity of the point $u=0$, which transforms system (11) to the form:

$$
\left\{\begin{array}{l}
x_{1}^{\prime}=-\omega_{0} x_{2}+\left(L_{1} x_{1}-\Omega_{1} x_{2}\right)\left(x_{1}^{2}+x_{2}^{2}\right)+o\left(r^{3}\right), \\
x_{2}^{\prime}=\omega_{0} x_{1}+\left(\Omega_{1} x_{1}+L_{1} x_{2}\right)\left(x_{1}^{2}+x_{2}^{2}\right)+o\left(r^{3}\right),
\end{array}\right.
$$

where $r=\sqrt{x_{1}^{2}+x_{2}^{2}}$. The number $L_{1}$ is called the first Lyapunov value of system (7) in the problem on the Andronov-Hopf bifurcation. In what follows, for simplicity, both numbers $L_{1}$ and $\Omega_{1}$ are called Laypunov values of system (7).

In a series of works, see, for instance, [1], [2, [15], [16], there were proposed various approaches and algorithms allowing one to calculate the Lyapunov values $L_{1}$ and $\Omega_{1}$ directly in terms of the initial equations. We provide a new scheme allowing us to calculate the Lyapunov values $L_{1}$ and $\Omega_{1}$ in terms of initial equation (7).

2.3.1. Auxiliary constructions. Since the matrix $A_{0}=A\left(\mu_{0}\right)$ has a pair of simple eigenvalues $\pm i \omega_{0}$, there exist non-zero vectors $e, g, e^{*}, g^{*} \in \mathbb{R}^{N}$ such that the identities

$$
A_{0}(e+i g)=i \omega_{0}(e+i g), \quad A_{0}^{*}\left(e^{*}+i g^{*}\right)=-i \omega_{0}\left(e^{*}+i g^{*}\right) .
$$

Here $A_{0}^{*}$ is the transposed matrix. The vectors $e, g, e^{*}, g^{*}$ can be normalized according the identities:

$$
\|e\|=\|g\|=1, \quad\left(e, e^{*}\right)=\left(g, g^{*}\right)=1,\left(e, g^{*}\right)=\left(g, e^{*}\right)=0 .
$$


In what follows we assume that the vectors $e, g, e^{*}, g^{*}$ are chose according identities (14). We let

$$
\begin{aligned}
& e(t)=e \cos 2 \pi t-g \sin 2 \pi t \\
& f_{3}(t)=b_{3}\left(e(t), \mu_{0}\right)+F_{2}(t) \int_{0}^{t} e^{-\tau T_{0} A_{0}} b_{2}\left(e(\tau), \mu_{0}\right) d \tau,
\end{aligned}
$$

where

$$
F_{2}(t)=T_{0} b_{2 x}^{\prime}\left(e(t), \mu_{0}\right) e^{T_{0} A_{0} t} .
$$

Here $b_{2 x}^{\prime}(x, \mu)$ is the Jacobi matrix of the vector function $b_{2}(x, \mu)$.

Finally, we define the vector:

$$
\rho_{3}=\int_{0}^{1} e^{(1-t) T_{0} A_{0}} f_{3}(t) d t .
$$

In what follows we shall make use of the following auxiliary statement. Let $y(t)$ be a continuous 1-periodic $N$-dimensional vector function. By $y_{c}$ and $y_{s}$ we denote the Fourier coefficients of this functions corresponding to $\cos 2 \pi t$ and $\sin 2 \pi t$. We define the vector

$$
u=\int_{0}^{1} e^{(1-t) T_{0} A_{0}} y(t) d t .
$$

Lemma 1. The identities

$$
\left(u, e^{*}\right)=\frac{1}{2}\left[\left(y_{c}, e^{*}\right)-\left(y_{s}, g^{*}\right)\right], \quad\left(u, g^{*}\right)=\frac{1}{2}\left[\left(y_{c}, g^{*}\right)+\left(y_{s}, e^{*}\right)\right]
$$

hold true.

This lemma can be proved by straightforward calculations with using identities (13).

2.3.2. Lyapunov values for two-dimensional systems. We first provide the scheme of obtaining Lyapunov values $L_{1}$ and $\Omega_{1}$ for the case when equation (7) is two-dimensional, that is, for the equation

$$
x^{\prime}=A(\mu) x+b(x, \mu), \quad x \in \mathbb{R}^{2} .
$$

Theorem 5. The Lyapunov values $L_{1}$ and $\Omega_{1}$ of two-dimensional system (21) are determined by the identities

$$
L_{1}=\left(\rho_{3}, e^{*}\right), \quad \Omega_{1}=-\left(\rho_{3}, g^{*}\right) .
$$

Numbers (22) are independent of the choice of the vectors $e, g, e^{*}, g^{*}$ according identities (14).

By Lemma 1 and identity (18) we obtain

Corollary 1. To calculate numbers (22), one can employ identities (20), in which one should let $u=\rho_{3}$ and $y(t)=f_{3}(t)$, where $f_{3}(t)$ is function (16).

2.3.3. Lyapunov values for case $N \geqslant 3$. We return back to system (7). Let $N \geqslant 3$ and $E_{0}$ be the eigenspace of the operator $A_{0}$ associated with the simple eigenvalues $\pm i \omega_{0}$. The space $E_{0}$ is two-dimensional; as its basis we can employ the vectors $e$ and $g$. The space $\mathbb{R}^{N}$ can be represented as $\mathbb{R}^{N}=E_{0} \oplus E^{0}$, where $E^{0}$ is an additional subspace of dimension $N-2$ invariant for $A_{0}$.

The identity $\mathbb{R}^{N}=E_{0} \oplus E^{0}$ defines the projectors $P_{0}: \mathbb{R}^{N} \rightarrow E_{0}$ and $P^{0}: \mathbb{R}^{N} \rightarrow E^{0}$ such that $P^{0}=I-P_{0}$ and the operator $P_{0}$ can be represented as

$$
P_{0} x=\left(x, e^{*}\right) e+\left(x, g^{*}\right) g ;
$$


the latter is implied by the fact that by the assumptions, the vectors $e, g, e^{*}, g^{*}$ according the identities (14). We let $B_{0}=e^{T_{0} A_{0}}$. It is easy to establish that the operator $I-B_{0}+P_{0}: \mathbb{R}^{N} \rightarrow$ $\mathbb{R}^{N}$ is invertible.

We define the vector and the matrix

$$
\rho_{2}=\int_{0}^{1} e^{(1-t) T_{0} A_{0}} b_{2}\left(e(t), \mu_{0}\right) d t, \quad B_{2}=\int_{0}^{1} e^{(1-t) T_{0} A_{0}} F_{2}(t) d t
$$

where $e(t)$ is function $(15)$ and $F_{2}(t)$ is matrix (17). We note that by construction, the inclusion $\rho_{2} \in E^{0}$ holds. Finally, we let

$$
\varphi=B_{2}\left(I-B_{0}+P_{0}\right)^{-1} \rho_{2} .
$$

Theorem 6. The Lyapunov values $L_{1}$ and $\Omega_{1}$ of system (7) are determined by the identities

$$
L_{1}=\left(\varphi+\rho_{3}, e^{*}\right), \quad \Omega_{1}=-\left(\varphi+\rho_{3}, g^{*}\right) .
$$

Numbers (26) are independent of the choice of the vectors $e, g, e^{*}, g^{*}$ according identities (14).

The analogue of Corollary 1 holds.

Corollary 2. To calculate numbers (26), one can employ identities (20), in which we should let $u=\varphi+\rho_{3}$ and $y(t)=g(t)+f_{3}(t)$, where $f_{3}(t)$ is function (16),

$$
g(t)=F_{2}(t)\left(I-B_{0}+P_{0}\right)^{-1} \rho_{2} .
$$

In an important particular case, when nonlinearity (3) involves no quadratic term, that is, $b_{2}(x, \mu) \equiv 0$, formulae $(26)$ are essentially simplified:

$$
L_{1}=\left(b_{0}, e^{*}\right), \quad \Omega_{1}=-\left(b_{0}, g^{*}\right),
$$

where $b_{0}=\int_{0}^{1} e^{(1-t) T_{0} A_{0}} b_{3}\left(e(t), \mu_{0}\right) d t$. At that, to calculate numbers 28, we can employ identities 20), in which we should let $u=b_{0}$ and $y(t)=b_{3}\left(e(t), \mu_{0}\right)$.

\subsubsection{Some properies of Andronov-Hopf bifurcation. We let}

$$
\gamma_{1}=\left(A^{\prime} e, e^{*}\right)+\left(A^{\prime} g, g^{*}\right), \quad \gamma_{2}=\left(A^{\prime} e, g^{*}\right)-\left(A^{\prime} g, e^{*}\right) ;
$$

here $A^{\prime}=A^{\prime}\left(\mu_{0}\right)$. One can show that numbers $(29)$ are independent of the choice of the vectors $e, g, e^{*}, g^{*}$ according identities (14).

Let $\gamma_{1} \neq 0$ and

$$
\mu_{2}=-\frac{2}{\gamma_{1}} L_{1}
$$

The results of work [10] imply the following statements.

Theorem 7. Let $\mu_{2}>0\left(\mu_{2}<0\right)$. Then the bifurcating solutions $x(t, \mu)$ to system (21) emerge as $\mu>\mu_{0}\left(\mu<\mu_{0}\right)$.

Theorem 8. Assume that all eigenvalues of the matrix $A_{0}$ not coinciding with $\pm \omega_{0} i$ have negative real parts. Then for all small $\left|\mu-\mu_{0}\right|$ the bifurcating solutions $x(t, \mu)$ to system (21) existing under the assumptions of Theorem 7 are asymptotically orbitally stable provided $L_{1}<0$. If $L_{1}>0$, they are unstable. 
2.3.5. Example: Langford model. As an illustration, we consider the Langford model (see, for instance, [15]):

$$
\left\{\begin{array}{l}
x_{1}^{\prime}=(2 \mu-1) x_{1}-x_{2}+x_{1} x_{3} \\
x_{2}^{\prime}=x_{1}+(2 \mu-1) x_{2}+x_{2} x_{3} \\
x_{3}^{\prime}=-\mu x_{3}-\left(x_{1}^{2}+x_{2}^{2}+x_{3}^{2}\right)
\end{array}\right.
$$

This system is of form (7) as $N=3$ and

$$
A(\mu)=\left[\begin{array}{ccc}
2 \mu-1 & -1 & 0 \\
1 & 2 \mu-1 & 0 \\
0 & 0 & -\mu
\end{array}\right], \quad b(x, \mu) \equiv b_{2}(x)=\left[\begin{array}{c}
x_{1} x_{3} \\
x_{2} x_{3} \\
-x_{1}^{2}-x_{2}^{2}-x_{3}^{2}
\end{array}\right] .
$$

As $\mu=\mu_{0}=1 / 2$, the matrix $A_{0}=A\left(\mu_{0}\right)$ has the eigenvalues $\lambda_{1,2}= \pm i$ and $\lambda_{3}=-1 / 2$. This is why the value $\mu=\mu_{0}$ is the Andronov-Hopf bifurcation point of system (31) and we have: $\omega_{0}=1$ and $T_{0}=2 \pi$. Let us calculate Lyapunov values $L_{1}$ and $\Omega_{1}$ of this system according identities (26).

As the eigenvectors $e, g, e^{*}, g^{*}$ satisfying identities (14), we choose the vectors

$$
e=e^{*}=\left[\begin{array}{l}
1 \\
0 \\
0
\end{array}\right], \quad g=g^{*}=\left[\begin{array}{c}
0 \\
-1 \\
0
\end{array}\right] \text {. }
$$

Since

$$
A^{\prime}=\left[\begin{array}{ccc}
2 & 0 & 0 \\
0 & 2 & 0 \\
0 & 0 & -1
\end{array}\right], \quad b_{2 x}^{\prime}(x)=\left[\begin{array}{ccc}
x_{3} & 0 & x_{1} \\
0 & x_{3} & x_{2} \\
-2 x_{1} & -2 x_{2} & -2 x_{3}
\end{array}\right]
$$

then, first, numbers (29) are equal: $\gamma_{1}=4$ and $\gamma_{2}=0$, second, we have:

$$
\begin{aligned}
& e^{T_{0} A_{0} t}=\left[\begin{array}{ccc}
\cos 2 \pi t & -\sin 2 \pi t & 0 \\
\sin 2 \pi t & \cos 2 \pi t & 0 \\
0 & 0 & e^{-\pi t}
\end{array}\right], \quad e(t)=\left[\begin{array}{c}
\cos 2 \pi t \\
\sin 2 \pi t \\
0
\end{array}\right], \\
& b_{2}(e(t)) \equiv\left[\begin{array}{c}
0 \\
0 \\
-1
\end{array}\right], \quad b_{2 x}^{\prime}(e(t))=\left[\begin{array}{ccc}
0 & \cos 2 \pi t \\
0 & 0 & \sin 2 \pi t \\
-2 \cos 2 \pi t & -2 \sin 2 \pi t & 0
\end{array}\right] .
\end{aligned}
$$

Finally, we have

$$
\left(I-B_{0}+P_{0}\right)^{-1}=\left[\begin{array}{ccc}
1 & 0 & 0 \\
0 & 1 & 0 \\
0 & 0 & \left(1-e^{-\pi}\right)^{-1}
\end{array}\right]
$$

where $B_{0}=e^{T_{0} A_{0}}$ and $P$ is the matrix of projector 23 .

We are in position to calculate functions (16) and (27). As a result we get

$$
f_{3}(t)=-2\left[\begin{array}{c}
\left(1-e^{-\pi t}\right) \cos 2 \pi t \\
\left(1-e^{-\pi t}\right) \sin 2 \pi t \\
0
\end{array}\right], \quad g(t)=-2\left[\begin{array}{c}
e^{-\pi t} \cos 2 \pi t \\
e^{-\pi t} \sin 2 \pi t \\
0
\end{array}\right] .
$$

Therefore, letting $y(t)=f_{3}(t)+g(t)$ and denoting by $y_{c}$ and $y_{s}$ the Fourier coefficients of the function corresponding to $\cos 2 \pi t$ and $\sin 2 \pi t$, we get:

$$
y(t)=-2\left[\begin{array}{c}
\cos 2 \pi t \\
\sin 2 \pi t \\
0
\end{array}\right], \quad y_{c}=\left[\begin{array}{c}
-2 \\
0 \\
0
\end{array}\right], \quad y_{s}=\left[\begin{array}{c}
0 \\
-2 \\
0
\end{array}\right]
$$

In view of Corollary 2, this implies: $\left(\varphi+\rho_{3}, e^{*}\right)=-2$ and $\left(\varphi+\rho_{3}, g^{*}\right)=0$. Thus, by (26) we finally get $L_{1}=-2$ and $\Omega_{1}=0$. 
We observe that here number $(30)$ is equal to $\mu_{2}=1$. This is why it follows from Theorems 7 and 8 that the bifurcating solutions to system (31) emerge as $\mu>1 / 2$ and they asymptotically orbitally stable.

\section{DiSCRETE DYNAMICAL SYSTEMS}

In this section we study the constructing of Lyapunov values for the discrete dynamical systems described by the equation:

$$
x_{n+1}=A(\mu) x_{n}+a\left(x_{n}, \mu\right)+u(\mu), x_{n} \in \mathbb{R}^{N}, \quad n=0,1,2, \ldots,
$$

where the matrix $A(\mu)$ and the function $a(x, \mu)$ are continuously differentiable in $x$ and $\mu$. We assume that the function $a(x, \mu)$ can be written as

$$
a(x, \mu)=a_{2}(x, \mu)+a_{3}(x, \mu)+\tilde{a}_{4}(x, \mu),
$$

where $a_{2}(x, \mu)$ and $a_{3}(x, \mu)$ are respectively quadratic and cubic in $x$ terms and $\tilde{a}_{4}(x, \mu)$ satisfies the relation: $\left\|\tilde{a}_{4}(x, \mu)\right\|=O\left(\|x\|^{4}\right), x \rightarrow 0$, uniformly in $\mu$. The function $u(\mu)$ is also supposed to be smooth and for some value $\mu=\mu_{0}$, the identity $u\left(\mu_{0}\right)=0$ holds. As $\mu=\mu_{0}$, system (32) has the equilibrium $x=0$.

3.1. Bifurcations and central manifold. If the matrix $A_{0}=A\left(\mu_{0}\right)$ has one or several eigenvalues with absolute values equal to 1 , then $\mu_{0}$ is a bifurcation point for system (32). In this case, as a rule, the phase portrait of system $(32)$ in the vicinity of the point $x=0$ transforms qualitatively as the parameter $\mu$ passes $\mu_{0}$.

As in the case of periodic dynamical systems, according the theorem on central manifold, the problem on local bifurcations for $N$-dimensional system (32) can be reduced to an equivalent (in the natural formulation) problem for a system of a lower dimension. In view of this, we provide some notions and facts to be used later.

Let the spectrum $\sigma$ of the matrix $A_{0}$ consists of two non-empty parts: $\sigma=\sigma_{0} \cup \sigma^{0}$, where $\sigma_{0}$ contains the eigenvalues with the absolute values equal to 1 , and $\sigma^{0}$ are other eigenvalues. We denote by $E_{0}$ and $E^{0}$ the root subspaces of the matrix $A_{0}$ corresponding, respectively, the parts $\sigma_{0}$ and $\sigma^{0}$ of its spectrum. Let $k_{0}$ and $k^{0}$ be the dimensions of the subspaces $E_{0}$ and $E^{0}$; then $k_{0}+k^{0}=N$ and $1 \leqslant k_{0}, k^{0} \leqslant N-1$. The space $\mathbb{R}^{N}$ is represented as the direct sum $\mathbb{R}^{N}=E_{0} \oplus E^{0}$ of the subspaces $E_{0}$ and $E^{0}$ invariant for $A_{0}: \mathbb{R}^{N} \rightarrow \mathbb{R}^{N}$. Finally, we denote by $P_{0}: \mathbb{R}^{N} \rightarrow E_{0}$ and $P^{0}: \mathbb{R}^{N} \rightarrow E^{0}$ the corresponding projectors.

According the theorem on central manifold, there exist a $\delta_{1}$-neighbourhood $T\left(0, \delta_{1}\right)$ of the point $x=0$ and a $\delta_{2}$-neighbourhood of the number $\mu_{0}$ such that as $\left|\mu-\mu_{0}\right|<\delta_{2}$ system (32) has a smooth invariant $k_{0}$-dimensional manifold $W(\mu)$ in the ball $T\left(0, \delta_{1}\right)$; this manifold contains $x=0$ and touches (as $\mu=\mu_{0}$ ) the subspace $E_{0}$ at the point $x=0$. The invariance of the manifold $W(\mu)$ for system (32) means that some its trajectory is located on the manifold $W(\mu)$, it stays on $W(\mu)$ for all other times until this trajectory stays in the ball $T\left(0, \delta_{1}\right)$. The manifold $W(\mu)$ is called central; it can be defined by the equation of form $v=\psi(u, \mu)$, where $u \in E_{0}, v \in E^{0}$ and the function $\psi(u, \mu)$ is smooth and satisfies the identities: $\psi\left(0, \mu_{0}\right)=0$, $\psi_{u}^{\prime}\left(0, \mu_{0}\right)=0$.

By projecting on the subspaces $E_{0}$ and $E^{0}$, in the vicinity of the point $x=0$, equation (32) can be represented as the system

$$
\left\{\begin{array}{l}
u_{n+1}=f\left(u_{n}, v_{n}, \mu\right) \\
v_{n+1}=g\left(u_{n}, v_{n}, \mu\right)
\end{array}\right.
$$

where $u_{n}=P_{0} x_{n}, v_{n}=P^{0} x_{n}$, and $f$ and $g$ are smooth functions taking values in $E_{0}$ and $E^{0}$, respectively, and same identities (5) as for continuous dynamical systems hold. 
Thus, the problem on local bifurcations in $N$-dimensional equation (32) can be reduced to studying the $k_{0}$-dimensional equation

$$
u_{n+1}=G\left(u_{n}, \mu\right), \quad u_{n} \in E_{0},
$$

where $G(u, \mu)=f(u, \psi(u, \mu), \mu)$. It involves all main features of bifurcation scenarios in initial equation (32). In particular, the analysis of equation (35) (usually by means of normal forms) leads one to Lyapunov values.

Here we consider the following main cases:

P1. The matrix $A_{0}$ has the simple eigenvale $1 ;$

P2. The matrix $A_{0}$ has the simple eigenvalue -1;

P3. The matrix $A_{0}$ has a pair of simple eigenvalues of $e^{ \pm i 2 \pi \theta_{0}}$, where $\theta_{0}$ is irrational $\theta_{0}=p / q$, where $p / q$ is a rational irreducible fraction and $q \geqslant 5$.

In all these cases we assume that the other eigenvalues of the matrix $A_{0}$ have absolute values not coinciding with 1.

We note that the case, when the matrix $A_{0}$ has a pair of pure simple eigenvalues of form $e^{ \pm i 2 \pi \theta}$, where $\theta=p / q$ is a irreducible fraction and $1 \leqslant q \leqslant 4$ is usually called strong resonance (see, for instance, [1]); we do not consider this case in the paper. We also note that Case P3, when $\theta_{0}=p / q$ and $q \geqslant 5$, is called weak resonance.

Finally, we note that while studying local bifurcation in Cases P2 and P3 and in some subcases of Case P1 one usually supposes that the function $u(\mu)$ in equation 32 vanishes, that is, this is the equation of the form

$$
x_{n+1}=A(\mu) x_{n}+a\left(x_{n}, \mu\right), \quad x_{n} \in \mathbb{R}^{N}, \quad n=0,1,2, \ldots
$$

3.2. Case P1: equilibria bifurcation. We consider first case P1. As in a similar Case S1 for autonomous equation (1), here the matter of the qualitative transformation of the behavior of system (32) in the vicinity of the point $x=0$ as the parameter $\mu$ passes through $\mu_{0}$ consists in emerging the non-zero equilibria. Such transformation of the behavior of equation (as for equation (1)) is called equilibria bifurcation of system (32).

In Case P1 equation (35) one-dimensional and by assumption (33) the function $G(u, \mu)$ at $\mu=\mu_{0}$ can be represented as

$$
G\left(u, \mu_{0}\right)=u+l_{1} u^{2}+l_{2} u^{3}+o\left(u^{3}\right) .
$$

In other words, as $\mu=\mu_{0}$, equation (35) is of the form

$$
u_{n+1}=u_{n}+l_{1} u_{n}^{2}+l_{2} u_{n}^{3}+o\left(u_{n}^{3}\right) .
$$

The numbers $l_{1}$ and $l_{2}$ are respectively called first and second Lyapunov value in the problem on equilibria bifurcation of system (32). We observe that Remark 1 also holds here up to an appropriate modification.

The equilibria bifurcation for discrete system (32) is similar to that for continuous system (2). This relates to the fact that the mentioned bifurcations are related with the emergence of non-zero equilibria in the vicinity of the point $x=0$ and the problem on such equilibria is reduced to the same equations:

$$
\begin{array}{ll}
A(\mu) x+b(x, \mu)+u(\mu)=0 & \text { for system (2), } \\
x=A(\mu) x+a(x, \mu)+u(\mu) & \text { for system } 32 .
\end{array}
$$

This is why all facts and statements in Section 2.2 hold also for discrete system (32) up to an appropriate modification.

Just to illustrate, we restrict ourselves by providing the analogue of Theorem 1. In other words, we provide a statement allowing us to calculate the Lyapunov values $l_{1}$ and $l_{2}$ of discrete system (32) for the mentioned bifurcation scenarios directly in terms of initial equation (32). 
By $e$ and $g$ we denote the eigenvectors of the matrix $A_{0}$ and the transposed matrix $A_{0}^{*}$, respectively, associated with the simple eigenvalue 1 . These vectors can be chosen in accordance with identities (9).

Theorem 9. Let the matrix $A_{0}$ has a simple eigenvalue 1 and its other eigenvalues have absolute values not coinciding with 1 . Then the first Lyapunov value of discrete system (32) in the problem on equilibria bifurcation is equal to $l_{1}=\left(a_{2}\left(e, \mu_{0}\right), g\right)$. If $a_{2}(x, \mu) \equiv 0$, then $l_{1}=0$ and $l_{2}=\left(a_{3}\left(e, \mu_{0}\right), g\right)$.

We note that here also Remarks 2 and 3 hold up to an appropriate modification.

As for continuous system (2), the equilibria bifurcation in system (32) can be realized as saddle-node bifurcation or as the transcritial bifurcation or pitchfork bifurcation. We note that two latter scenarios require the zero function $u(\mu)$ in equation (32), that is, this equation should be of form (36). Finally, we note that Theorems 24 remain true for discrete system 32 up to an appropriate modification.

In conclusion of this subsection, as an illustration, we provide model examples of the mentioned bifurcation scenarios.

A model example of saddle-node bifurcation is given by the scalar equation $x_{n+1}=x_{n}+\mu-x_{n}^{2}$. As $\mu<0$, this equation has no equilibria, as $\mu=0$, it has only the equilibrium $x=0$, while as $\mu>0$, it has two non-zero equilibria $x= \pm \sqrt{\mu}$. Thus, as $\mu$ passes through $\mu=0$, in the vicinity of the point $x=0$, there emerges first the single equilibrium $x=0$ (as $\mu>0$ ) for the considered equation and then (as $\mu>0$ ) it "splits" into two non-zero equilibria $x_{1,2}= \pm \sqrt{\mu}$; the one of them is stable while the other is not. Here the first Lyapunov value is equal to $l_{1}=-1$.

A model example of the transcritical bifurcation is given by the scalar equation $x_{n+1}=$ $\mu x_{n}-x_{n}^{2}$. For all $\mu$ this equation has the equilibrium $x=0$. As $\mu$ passes the value $\mu=1$, in the vicinity of the point $x=0$, there arises the non-zero equilibrium $x=\mu-1$ for this equation and this equilibrium is stable as $\mu>1$ and unstable as $\mu<1$. Here we also have $l_{1}=-1$.

A model example of pitchfork bifurcation is given by the scalar equation $x_{n+1}=\mu x_{n}-x_{n}^{3}$. It also has the equilibrium $x=0$ for all $\mu$. As $\mu<1$, this equation has no other equilibria, while as $\mu$ passes the value $\mu=1$, in the vicinity of the point $x=0$ there arise two non-zero equilibria $x= \pm \sqrt{\mu-1}$, which are stable. Here we have $l_{1}=0$ and $l_{2}=-1$.

3.3. Bifurcation of doubling period. We consider equation $(36)$, in which $a(x, \mu)$ is determined by identity (33). Assume that Case P2 holds. Then the main bifurcation scenarion in the vicinity of the point $x=0$ is the bifurcation of doubling period.

A model example of bifurcation of doubling period is given by the scalar equation $x_{n+1}=$ $\mu x_{n}+x_{n}^{3}$. For all $\mu$ it has equilibrium $x=0$. As $|\mu|<1$, this point is stable while as $\mu<-1$ and $\mu>1$, it is unstable. As $\mu$ passes the value $\mu=-1$, in the vicinity of the point $x=0$ there arises a stable cycle of period 2: $x_{1}=\sqrt{-1-\mu}, x_{2}=-\sqrt{-1-\mu}$. The scenario of such kind is called bifurcation of doubling period. We also observe that in this example, the point $\mu=1$ is a pitchfork bifurcation.

As $N \geqslant 2$, the bifurcation of doubling period follows the same scenario.

Since the matrix $A_{0}$ has the simple eigenvalue -1 and has no other eigenvalues with absolute value equal to 1 , then equation (35) is one-dimensional and by assumption (33), as $\mu=\mu_{0}$, the function $G(u, \mu)$ can be represented as (see, for instance, [1]):

$$
G\left(u, \mu_{0}\right)=-u-l_{1} u^{3}+o\left(u^{3}\right) .
$$

The number $l_{1}$ is called first Lyapunov value for the bifurcation of doubling period in system (36).

Let us provide a statement allowing us to calculate the Lyapunov value $l_{1}$ directly in terms of initial equation (36). In order to do this, by $e$ and $g$ we denote the eigenvectors of matrix 
$A_{0}$ and of the transposed matrix $A_{0}^{*}$, respectively, associated with the eigenvalue -1 . These vectors can be chosen according identities (9). The subspace $E_{0}$ is one-dimensional and it contained the vector $e$. Finally, the projectors $P_{0}: \mathbb{R}^{N} \rightarrow E_{0}$ and $P^{0}: \mathbb{R}^{N} \rightarrow E^{0}$ can be defined by the identities: $P_{0} x=(x, g) e$ and $P^{0}=I-P_{0}$. It is easy to establish that the operator $I-A_{0}^{2}+P_{0}: \mathbb{R}^{N} \rightarrow \mathbb{R}^{N}$ is invertible.

To simplify the notations, we denote $a_{2}=a_{2}\left(e, \mu_{0}\right), a_{3}=a_{3}\left(e, \mu_{0}\right)$ and $a_{2}^{\prime}=a_{2 x}^{\prime}\left(e, \mu_{0}\right)$.

Theorem 10. Assume that the matrix $A_{0}=A\left(\mu_{0}\right)$ has the simple eigenvalue -1 and its other eigenvalues have the absolute values not coinciding with 1 . Then the first Lyapunov value $l_{1}$ for the bifurcation of doubling period for system (36) is equal to

$$
l_{1}=-\frac{\left(2 a_{3}+a_{2}^{\prime}\left[a_{2}+\left(I+A_{0}\right) e_{1}\right], g\right)}{2}
$$

where $e_{1}=\left(I-A_{0}^{2}+P_{0}\right)^{-1}\left(I+A_{0}\right) a_{2}$.

Remark 4. Number (37) is independent of the normalization of the vectors e and $g$ according identities (9). Indeed, as we have mentioned above (see Remark 3), these options differ by the sign. It is easy to see that in formula (37) both options leads one to the same number.

We consider an important particular case, when system (36) is scalar, namely, we consider the equation

$$
x_{n+1}=\beta_{1}(\mu) x_{n}+\beta_{2}(\mu) x_{n}^{2}+\beta_{3}(\mu) x_{n}^{3}+O\left(x_{n}^{4}\right), \quad x_{n} \in \mathbb{R}^{1},
$$

in which the functions $\beta_{j}(\mu)$ are smooth and $\beta_{1}\left(\mu_{0}\right)=-1$. In this case formula (37) is simplified

$$
l_{1}=-\left(\beta_{2}^{2}+\beta_{3}\right)
$$

where $\beta_{2}=\beta_{2}\left(\mu_{0}\right)$ and $\beta_{3}=\beta_{3}\left(\mu_{0}\right)$.

3.3.1. Properties of bifurcation of doubling period. We provide some properties of bifurcation of doubling period in equation (36) implied by the results of work [10].

Theorem 11. Suppose that under the assumptions of Theorem 10 the relations

$$
l_{1} \neq 0, \quad \gamma_{1}=\left(A^{\prime}\left(\mu_{0}\right) e, g\right) \neq 0
$$

hold. Let $\mu_{2} \equiv l_{1} / \gamma_{1}>0$. Then there exists $\delta>0$ such that

1. As $\mu \in\left(\mu_{0}-\delta, \mu_{0}\right]$, equation (36) has the unique equilibrium $x=0$ in the $\delta$-neighbourhood of the point $x=0$ and has no cycles, while for each $\mu \in\left(\mu_{0}, \mu_{0}+\delta\right)$, apart of the equilibrium $x=0$, it has one non-zero cycle of period 2: $x_{1}=x_{1}(\mu), x_{2}=x_{2}(\mu)$.

2. The functions $x_{1}(\mu)$ and $x_{2}(\mu)\left(x_{1}\left(\mu_{0}\right)=x_{2}\left(\mu_{0}\right)=0\right)$ defined for $\mu \in\left[\mu_{0}, \mu_{0}+\delta\right)$ are continuously differentiable and as $\mu=\mu_{0}$, the eigenvector of the matrix $A_{0}$ associated with the eigenvalue -1 is tangent to their graphs.

3. Let $l_{1}<0\left(l_{1}>0\right)$ and let the eigenvalues of the matrix $A_{0}=A\left(\mu_{0}\right)$ not coinciding with -1 have the absolute values less than 1 . Then the cycle $x_{1}=x_{1}(\mu), x_{2}=x_{2}(\mu)$ is asymptotically stable (unstable) as $\mu \in\left(\mu_{0}, \mu_{0}+\delta\right)$.

A similar statement can be provided in the case $\mu_{2}<0$. In this case only the direction of the bifurcation changes, that is, the bifurcating solutions emerge as $\mu \in\left(\mu_{0}-\delta, \mu_{0}\right)$.

We also note that for scalar equation (38) introduced by the second identity in (40), the number $\gamma_{1}$ is $\gamma_{1}=\beta_{1}^{\prime}\left(\mu_{0}\right)$. 
3.3.2. Example: Henon model. As an example we consider the Henon model (see, for instance, [1]):

$$
\left\{\begin{array}{l}
u_{n+1}=v_{n}, \\
v_{n+1}=a-\mu u_{n}-v_{n}^{2},
\end{array}\right.
$$

in which $0<a<3$ and $-1<\mu<1$. In what follows the value $a$ is fixed, while $\mu$ is regarded as the bifurcating parameter.

System (41) possesses the equilibrium $\left(u^{*}(\mu), v^{*}(\mu)\right)$, where

$$
u^{*}(\mu)=v^{*}(\mu)=\frac{-(1+\mu)+\sqrt{(1+\mu)^{2}+4 a}}{2} .
$$

Making the change $u=x+u^{*}(\mu)$ and $v=y+v^{*}(\mu)$ in (41), we pass to the system:

$$
\left\{\begin{array}{l}
x_{n+1}=y_{n}, \\
y_{n+1}=-\mu x_{n}-2 u^{*}(\mu) y_{n}-y_{n}^{2},
\end{array}\right.
$$

that is, to system of form $(36)$ as $N=2$ with

$$
A(\mu)=\left[\begin{array}{cc}
0 & 1 \\
-\mu & -2 u^{*}(\mu)
\end{array}\right], \quad a(w, \mu)=a_{2}(w)=\left[\begin{array}{c}
0 \\
-y^{2}
\end{array}\right]
$$

here $w=(x, y)$. As $\mu=\mu_{0}=2 \sqrt{a / 3}-1$, the matrix $A(\mu)$ has the eigenvalues $\lambda_{1}=-1$ and $\lambda_{2}=-\mu_{0}$. Hence, we can expect that as the parameter $\mu$ passes the value $\mu=\mu_{0}$, in the vicinity of the equilibrium $\left(u^{*}(\mu), v^{*}(\mu)\right)$ of system (41), the cycles of period 2 emerge. Let us study this issue.

Let us find the eigenvectors $e$ and $g$ of the matrix

$$
A_{0}=A\left(\mu_{0}\right)=\left[\begin{array}{cc}
0 & 1 \\
-\mu_{0} & -\left(1+\mu_{0}\right)
\end{array}\right]
$$

and of the transposed matrix $A_{0}^{*}$ associated with the eigenvalue -1 and satisfying the identities $\|e\|=1$ and $(e, g)=1$. We have

$$
e=\frac{1}{\sqrt{2}}\left[\begin{array}{c}
1 \\
-1
\end{array}\right], \quad g=\frac{\sqrt{2}}{\mu_{0}-1}\left[\begin{array}{c}
\mu_{0} \\
1
\end{array}\right] .
$$

Let us calculate the expression in formula (37). We have

$$
\begin{aligned}
& a_{2}=-\frac{1}{2}\left[\begin{array}{l}
0 \\
1
\end{array}\right], \quad a_{2}^{\prime}=\left[\begin{array}{cc}
0 & 0 \\
0 & \sqrt{2}
\end{array}\right], \quad P_{0}=\frac{1}{\mu_{0}-1}\left[\begin{array}{cc}
\mu_{0} & 1 \\
-\mu_{0} & -1
\end{array}\right], \\
& \left(I-A_{0}^{2}+P_{0}\right)^{-1}=-\frac{1}{\left(1-\mu_{0}\right)^{2}\left(1+\mu_{0}\right)}\left[\begin{array}{cc}
-\mu_{0}^{3}+\mu_{0}-1 & -\mu_{0}^{2} \\
\mu_{0}^{3} & \mu_{0}^{2}+\mu_{0}-1
\end{array}\right], \\
& e_{1}=\frac{1}{2\left(\mu_{0}^{2}-1\right)}\left[\begin{array}{c}
1 \\
-\mu_{0}
\end{array}\right], \quad a_{3}=0 .
\end{aligned}
$$

We substitute these expression into formula (37) and we get

$$
l_{1}=\frac{1}{2\left(\mu_{0}^{2}-1\right)},
$$

that is, in the considered problem the first Lyapunov value is negative.

Since

$$
A^{\prime}\left(\mu_{0}\right)=\left[\begin{array}{cc}
0 & 0 \\
-1 & 1 / 2
\end{array}\right]
$$


then the number $\gamma_{1}$ defined by the second identity in 40 is $\gamma_{1}=\frac{3}{2\left(1-\mu_{0}\right)}$. Then

$$
\mu_{2} \equiv l_{1} / \gamma_{1}=-\frac{1}{3\left(\mu_{0}+1\right)}<0 .
$$

By Theorem 11 this implies that the cycles of period 2 emerge in the vicinity of the equilibrium $\left(u^{*}(\mu), v^{*}(\mu)\right)$ of system (41) as $\mu<\mu_{0}$ and they are asymptotically stable.

3.4. Andronov-Hopf bifurcation. We continue considering equation (36). We suppose that Case P3 holds, that is, we assume that the matrix $A_{0}$ has a pair of simple eigenvalues of form $e^{ \pm i 2 \pi \theta_{0}}$, where $\theta_{0}$ is irrational or $\theta_{0}=p / q$, where $p / q$ is rational irreducible fraction and $q \geqslant 5$. For the sake of simplicity we assume that $N=2$, that is, equation (36) is twodimensional. Namely, we assume that it is of the form:

$$
x_{n+1}=A(\mu) x_{n}+a\left(x_{n}, \mu\right), \quad x_{n} \in \mathbb{R}^{2}, n=0,1,2, \ldots,
$$

and we assume that the matrix $A(\mu)$ is of the form

$$
A(\mu)=(1+\varphi(\mu))\left[\begin{array}{cc}
\cos 2 \pi\left(\theta_{0}+\psi(\mu)\right) & -\sin 2 \pi\left(\theta_{0}+\psi(\mu)\right) \\
\sin 2 \pi\left(\theta_{0}+\psi(\mu)\right) & \cos 2 \pi\left(\theta_{0}+\psi(\mu)\right)
\end{array}\right],
$$

where the functions $\varphi(\mu)$ and $\psi(\mu)$ are smooth and satisfy the identities: $\varphi\left(\mu_{0}\right)=0$ and $\psi\left(\mu_{0}\right)=0$, and at that, $\varphi^{\prime}\left(\mu_{0}\right) \neq 0$ and $\psi^{\prime}\left(\mu_{0}\right) \neq 0$.

In the considered case the main scenario of local bifurcation in the vicinity of the equilibrium $x=0$ of equation (42) as the parameter $\mu$ passes through $\mu_{0}$ is the emergence of an invariant curve $\gamma(\mu)$ in the vicinity of the point $x=0$ bordering the attraction or repulsion basin of this point. Similar to the continuous case considered in Subsection 2.3, such scenario is called Andronov-Hopf bifurcation, see, for instance, [1]. The dynamics of system (42) on the mentioned invariant curve can turn out to be very complicated and can contain a family of periodic and quasi-periodic orbits.

As a rule, for small $\left|\mu-\mu_{0}\right|$, the invariant curve $\gamma(\mu)$ of equation (42) emerges in of the three cases: (S1), $\mu>\mu_{0}$; (S2), $\mu<\mu_{0}$; (S3), $\mu=\mu_{0}$. The latter case is called degenerate; it is typical for linear and conservative systems. The first two cases hold under some non-degeneracy condition for nonlinear term (33) in the right hand side of equation (42) (one of the versions of such condition will be given below). Under this condition, in cases (S1) and (S2), to each $\mu$, exactly one invariant curve $\gamma(\mu)$ corresponds and the function $\gamma(\mu)$ depends smoothly on $\mu$ and it contracts to the point $x=0$ as $\mu \rightarrow \mu_{0}$.

In the considered problem equation (35) is two-dimensional. Due to identity (33), by means of the theory of normal forms, this equation as $\mu=\mu_{0}$ can be represented as (see, for instance, [1]):

$$
\left\{\begin{array}{l}
x_{n+1}=x_{n} \cos 2 \pi \theta_{0}-y_{n} \sin 2 \pi \theta_{0}+\left(\alpha x_{n}-\beta y_{n}\right)\left(x_{n}^{2}+y_{n}^{2}\right)+o\left(r_{n}^{3}\right), \\
y_{n+1}=x_{n} \sin 2 \pi \theta_{0}+y_{n} \cos 2 \pi \theta_{0}+\left(\beta x_{n}+\alpha y_{n}\right)\left(x_{n}^{2}+y_{n}^{2}\right)+o\left(r_{n}^{3}\right),
\end{array}\right.
$$

where $r_{n}=\sqrt{x_{n}^{2}+y_{n}^{2}}$. We let

$$
L_{1}=\alpha \cos 2 \pi \theta_{0}+\beta \sin 2 \pi \theta_{0}, \quad \Omega_{1}=\beta \cos 2 \pi \theta_{0}-\alpha \sin 2 \pi \theta_{0} .
$$

The number $L_{1}$ is called the first Lyapunov value of system 42 in the problem on AndronovHopf bifurcation. For the sake of simplicity, in what follows, we call both numbers $L_{1}$ and $\Omega_{1}$ Lyapunov values of system (42).

Let us provide a new scheme allowing one to calculate Lyapunov values $L_{1}$ and $\Omega_{1}$ in terms of initial equation (42) in the case, when the nonlinearity (33) begins with the cubic term, that is, it is of the form:

$$
a(x, \mu)=a_{3}(x, \mu)+\tilde{a}_{4}(x, \mu) .
$$

We let

$$
\chi(\varphi)=\left(a_{3}\left(e(\varphi), \mu_{0}\right), h(\varphi)\right), \quad \psi(\varphi)=\left(a_{3}\left(g(\varphi), \mu_{0}\right), h(\varphi)\right),
$$


where

$$
e(\varphi)=\left[\begin{array}{c}
\cos \varphi \\
\sin \varphi
\end{array}\right], \quad g(\varphi)=\left[\begin{array}{c}
\sin \varphi \\
-\cos \varphi
\end{array}\right], \quad h(\varphi)=\left[\begin{array}{c}
\cos \left(\varphi+2 \pi \theta_{0}\right) \\
\sin \left(\varphi+2 \pi \theta_{0}\right)
\end{array}\right]
$$

Theorem 12. The Lyapunov values $L_{1}$ and $\Omega_{1}$ for Andronov-Hopf bifurcation in system (36) are equal to

$$
L_{1}=\frac{1}{2 \pi} \int_{0}^{2 \pi} \chi(\varphi) d \varphi, \quad \Omega_{1}=\frac{1}{2 \pi} \int_{0}^{2 \pi} \psi(\varphi) d \varphi .
$$

\section{NON-AUTONOMOUS PERIODIC EQUATIONS}

In this section we study the issue on constructing Lyapunov values for dynamical systems described by non-autonomous differential equation with a $T$-periodic in $t$ right hand side:

$$
x^{\prime}=A(t, \mu) x+a(x, t, \mu)+g(t, \mu), \quad x \in \mathbb{R}^{N},
$$

in which the matrix $A(t, \mu)$ and the functions $a(x, t, \mu)$ and $g(t, \mu)$ are continuous in $t$ and continuous differentiable in $x$ and $\mu$. We assume that the function $a(x, t, \mu)$ can be represented as

$$
a(x, t, \mu)=a_{2}(x, t, \mu)+a_{3}(x, t, \mu)+\tilde{a}_{4}(x, t, \mu),
$$

where $a_{2}(x, t, \mu)$ and $a_{3}(x, t, \mu)$ involves, respectively, the quadratic and cubic in $x$ terms and the nonlinearity $\tilde{a}_{4}(x, t, \mu)$ satisfies the relation: $\left\|\tilde{a}_{4}(x, t, \mu)\right\|=O\left(\|x\|^{4}\right), x \rightarrow 0$, uniforlmy in $t$ and $\mu$. The function $g(t, \mu)$ vanishes for some value $\mu=\mu_{0}: g\left(t, \mu_{0}\right) \equiv 0$. As $\mu=\mu_{0}$, system (45) has the equilibrium $x=0$.

If some $\mu=\mu_{0}$ the linear $T$-periodic system

$$
x^{\prime}=A(t, \mu) x, \quad x \in \mathbb{R}^{N},
$$

has several multiplicators with absolute value equal to 1 , then $\mu_{0}$ is the bifurcation point of system (45). In this case, as the parameter $\mu$ passes through $\mu_{0}$, the behavior of system (45) in the vicinity of the point $x=0$ usually transforms qualitatively.

Here we consider the following main cases:

S1. System (46) has the simple multiplicator 1;

S2. System (46) has the pair of simple multiplications of form $e^{ \pm i 2 \pi \theta_{0}}$, where $\theta_{0}$ is an irrational number or $\theta_{0}$ is a rational number of form $\theta_{0}=p / q$, where $p / q$ is a irreducible fraction and $q \geqslant 5$.

At that we suppose that the other multiplicators of system (46) have absolute values more or less than 1.

We observe that system (46) can not have the simple multiplicator -1 . As for discrete system (32), the case, when system (46) has the pair of simple multiplicators of form $e^{ \pm i 2 \pi \theta}$, where $\theta=p / q$ is a irreducible fraction and $1 \leqslant q \leqslant 4$, is called the strong resonance; we do not consider this case the present paper. Case S2, when $\theta_{0}=p / q$ and $q \geqslant 5$, is usually called weak resonance.

4.1. Passage to discrete equation. The problem on local bifurcations of system 45 is equivalent in the natural sense to the problem on local bifurcations of discrete dynamical system

$$
x_{n+1}=U\left(x_{n}, \mu\right), \quad n=0,1,2, \ldots,
$$

where $x_{n} \in \mathbb{R}^{N}, U(*, \mu): \mathbb{R}^{N} \rightarrow \mathbb{R}^{N}$, is the translation operator, (see, for instance, [17]) along the trajectories of system (45) in time from 0 to $T$. The operator $U(*, \mu)$ called also Poincaré mapping can be represented as

$$
U(x, \mu)=V(\mu) x+v(x, \mu)+u(\mu),
$$


where $V(\mu)$ is the monodromy matrix of linear system 46); the function $u(\mu)$ satisfies the condition $u\left(\mu_{0}\right)=0 ; v(x, \mu)$ is a nonlinear operator, which can be represented as

$$
v(x, \mu)=v_{2}(x, \mu)+v_{3}(x, \mu)+\tilde{v}_{4}(x, \mu),
$$

where $v_{2}(x, \mu)$ and $v_{3}(x, \mu)$ involve, respectively, quadratic and cubic in $x$ terms, while the nonlinearity $\tilde{v}_{4}(x, \mu)$ satisfies the relation: $\left\|\tilde{v}_{4}(x, \mu)\right\|=O\left(\|x\|^{4}\right), x \rightarrow 0$, uniformly in $\mu$.

We note that the equilibria of equation (47) determine the initial values of $T$-periodic solutions to system (45), and each point of $q$-cycle of equation (47) determines initial values of $q T$-periodic solutions to this system.

The functions involved in (48) can be found explicitly provided, for instance, we know the fundamental matrix $X(t, \mu)$ of solutions of linear system (46) obeying the initial condition $X(0, \mu)=I$. Then $V(\mu)=X(T, \mu)$ and, for instance,

$$
\begin{aligned}
& v_{2}(x, \mu)=V(\mu) \int_{0}^{T} X^{-1}(\tau, \mu) a_{2}(X(\tau, \mu) x, \tau, \mu) d \tau, \\
& u(\mu)=V(\mu) \int_{0}^{T} X^{-1}(\tau, \mu) g(\tau, \mu) d \tau .
\end{aligned}
$$

In particular, if $A(t, \mu)$ is a constant in $t$ matrix, that is, $A(t, \mu) \equiv A_{0}(\mu)$, then $X(t, \mu)=e^{T A_{0}(\mu)}$.

The eigenvalues of the matrix $V(\mu)$ are the multiplicators of linear system (46). This is why here Cases S1 and S2 for differential equation (45) correspond to Cases P1 and P3 for discrete system (47). Thus, the problem on Lyapunov values for differential equation (45) can be reduced to a similar problem for discrete system (47). To study the latter, we can employ the scheme proposed in the previous section. At that, the Lyapunov values for differential equation (45) will be determined as Lyapunov values of system (47).

4.2. Main bifurcation scenarios. First we consider Case S1. This case corresponds to Case P1 for discrete system (47). As it was mentioned above, in this case the matter of the qualitative transformation of the behavior of system (47) as the parameter $\mu$ passes through $\mu_{0}$ is the emergence of non-zero equilibria in the vicinity of the point $x=0$. But the equilibria of equation (47) define initial values of $T$-periodic solutions of system (45). This is why in Case S1 the main scenario of the transformation of the behavior of system (45) is the emergence of nonzero $T$-periodic solutions of small amplitude in the vicinity of the point $x=0$. Such scenario is usually called the bifurcation of forced oscillations in system (45). In its turn, this bifurcation can realizes as the saddle-node bifurcation or transcritial bifurcation or pitchfork bifurcation.

Suppose that Case S2 holds and for simplicity, let $N=2$. This case corresponds Case P3 for discrete system (47). As it has been mentioned above, in this case the qualitative transformation of the behavior of system (47) as the parameter $\mu$ passes $\mu_{0}$ consists in the emergence of the invariant curve $\gamma(\mu)$ in the vicinity of the point $x=0$. This corresponds to the fact that in the space $\mathbb{R}^{2} \times \mathbb{R}^{1}$ (where $x \in \mathbb{R}^{2}$ and $t \in \mathbb{R}^{1}$ ) there emerges a smooth two-dimensional surface $\Upsilon(\mu)$ involving the axis $t$ and being invariant for differential equation (45). The dynamics of system 45 on the surface $\Upsilon(\mu)$ can be very complicated and can include a family of periodic and quasiperiodic solutions.

4.3. Lyapunov values. For each of the mentioned bifurcation scenarios in system (45), the issues on calculating Lyapunov values and the properties of the bifurcations can be solved by the scheme exposed in the previous section. Just to illustrate, we restrict ourselves by providing an analogue of Theorem 9. Namely, we provide a statement allowing us to calculate the first Lyapunov value for differential equation 45 in Case S1.

We denote by $e$ and $g$ the eigenvectors of the matrix $V_{0}=V\left(\mu_{0}\right)$ and of the transposed matrix $V_{0}^{*}$, respectively, associated with the simple eigenvalue 1 . These vectors can be chosen according identities (9). 
Theorem 13. Assume that Case S1 holds. Then the first Lyapunov value of system (45) in the problem of forced oscillations is equal to

$$
l_{1}=\int_{0}^{T}\left(X^{-1}\left(\tau, \mu_{0}\right) a_{2}\left(X\left(\tau, \mu_{0}\right) e, \tau, \mu_{0}\right), g\right) d \tau .
$$

In an important particular case when the matrix $A\left(t, \mu_{0}\right)$ is constant, that is, $A\left(t, \mu_{0}\right) \equiv A_{0}$, formula 49) becomes very simple:

$$
l_{1}=\int_{0}^{T}\left(a_{2}\left(e, \tau, \mu_{0}\right), g\right) d \tau .
$$

In this formula $e$ and $g$ are the eigenvectors of the matrices $A_{0}$ and $A_{0}^{*}$, respectively, associated with the simple eigenvalue 0 and obeying identities $(9)$.

4.3.1. Example. As an illustration, we consider the scalar equation

$$
x^{\prime}=\mu(1+\cos t) x+x^{2} .
$$

This equation is of form (45) as $A(t, \mu)=\mu(1+\cos t), a(x, t, \mu) \equiv a_{2}(x)=x^{2}$ and $g(t, \mu) \equiv 0$. The value $\mu=0$ is the bifurcation point for this equation and at that, Case S1 holds, that is, as the parameter $\mu$ passes the value $\mu=0$, in the vicinity of the point $x=0$, the bifurcation of forced oscillations hold for equation (51). Since in the considered example $g(t, \mu) \equiv 0$ and the nonlinearity involves $a(x, t, \mu)$ involves only quadratic terms, the bifurcation realizes as the transcritical one.

Let us calculate the first Lyapunov value $l_{1}$ for equation (51). Here we can employ formula (50). As $e$ and $g$, we can take the numbers $e=1$ and $g=1$. Since $T=2 \pi$, we get

$$
l_{1}=\int_{0}^{2 \pi}\left(a_{2}(e), g\right) d \tau=2 \pi .
$$

To study the properties of bifurcation in equation (51), we proceed to the discrete model of form (47). Here the operator $V(\mu)$ is the function

$$
V(\mu)=\exp \left(\mu \int_{0}^{2 \pi}(1+\cos \tau) d \tau\right)=e^{2 \pi \mu}
$$

Then we employ Theorem 3 , more precisely, by its analogue for discrete systems. Here the second number in (10) is obviously equal to $\gamma_{1}=\left(V^{\prime}(0) e, g\right)=2 \pi>0$. This is why the mentioned theorem implies the emerging $2 \pi$-periodic solutions of equation (51) are asymptotically stable as $\mu>0$ and unstable as $\mu<0$.

\section{Proof of MAin STATEMENTS}

5.1. Proof of Theorem 1. Under the assumptions of this theorem, subspace $E_{0}$ is onedimensional and the projector $P_{0}: \mathbb{R}^{N} \rightarrow E_{0}$ can be defined by the identity $P_{0} x=(x, g) e$. This is why here equation (6) is also one-dimensional, namely, as $\mu=\mu_{0}$, it is of form:

$$
u^{\prime}=P_{0} b_{2}\left(u+\psi\left(u, \mu_{0}\right), \mu_{0}\right)+P_{0} b_{3}\left(u+\psi\left(u, \mu_{0}\right), \mu_{0}\right)+P_{0} b_{4}\left(u+\psi\left(u, \mu_{0}\right), \mu_{0}\right) .
$$

By the identities $\psi\left(0, \mu_{0}\right)=\psi_{u}^{\prime}\left(0, \mu_{0}\right)=0$ this implies the statement of the theorem. 
5.2. Proof of Theorem 5 and 6. Here we restrict ourselves by proving Theorem 5 . Theorem 6 can be proved by the same schemes but it requires more bulky constructions.

Without loss of generality we can assume that as $\mu=\mu_{0}$, equation (21) is of form

$$
x^{\prime}=A x+a_{2}(x)+a_{3}(x)+\ldots, \quad x \in \mathbb{R}^{2},
$$

where

$$
\begin{aligned}
& A=\left[\begin{array}{cc}
0 & 1 \\
-1 & 0
\end{array}\right], \quad x=\left[\begin{array}{l}
x_{1} \\
x_{2}
\end{array}\right], \\
& a_{2}(x)=\left[\begin{array}{c}
a_{20} x_{1}^{2}+a_{11} x_{1} x_{2}+a_{02} x_{2}^{2} \\
b_{20} x_{1}^{2}+b_{11} x_{1} x_{2}+b_{02} x_{2}^{2}
\end{array}\right], \\
& a_{3}(x)=\left[\begin{array}{c}
a_{30} x_{1}^{3}+a_{21} x_{1}^{2} x_{2}+a_{12} x_{1} x_{2}^{2}+a_{03} x_{2}^{3} \\
b_{30} x_{1}^{3}+b_{21} x_{1}^{2} x_{2}+b_{12} x_{1} x_{2}^{2}+b_{03} x_{2}^{3}
\end{array}\right] .
\end{aligned}
$$

The eigenvectors $e+i g$ and $e^{*}+i g^{*}$ of the matrices $A$ and $A^{*}$ chosen in accordance with identities (14) are determined by the identities:

$$
e=e^{*}=\left[\begin{array}{l}
1 \\
0
\end{array}\right], \quad g=g^{*}=\left[\begin{array}{l}
0 \\
1
\end{array}\right] .
$$

Functions (15) and (16) cast into the form:

$$
e(t)=\left[\begin{array}{c}
\cos 2 \pi t \\
-\sin 2 \pi t
\end{array}\right], \quad f_{3}(t)=a_{3}(e(t))+F_{2}(t) \int_{0}^{t} e^{-T_{0} A \tau} a_{2}(e(\tau)) d \tau,
$$

where $T_{0}=2 \pi, F_{2}(t)=T_{0} a_{2 x}^{\prime}(e(t)) e^{T_{0} A t}$; here $a_{2 x}^{\prime}(x)$ is the Jacobi matrix of the vector function $a_{2}(x)$.

To prove Theorem 5, we need to show that the numbers defined by identities (22),

$$
\Delta_{0}=\left(\rho_{3}, e^{*}\right), \quad \Delta_{1}=-\left(\rho_{3}, g^{*}\right),
$$

where $\rho_{3}$ is vector 18$)$ coincide with the Lyapunov values $L_{1}$ and $\Omega_{1}$. We restrict ourselves by checking the identity $\Delta_{0}=L_{1}$.

By Corollary 1 we obtain that to find the number $\Delta_{0}$, we should let $y(t)=f_{3}(t)$ in formulae (19) and (20). We split calculation of the Fourier coefficients $y_{c}$ and $y_{s}$ of the function $y(t)=$ $f_{3}(t)$ involved in 20 into two steps.

5.2.1. First step. At the first step we let $a_{2}(x) \equiv 0$. Then $f_{3}(t)=a_{3}(e(t))$. By (54) we have

$$
\begin{aligned}
a_{3}(e(t))= & \frac{1}{4} \cos 2 \pi t\left[\begin{array}{c}
3 a_{30}+a_{12} \\
3 b_{30}+b_{12}
\end{array}\right]-\frac{1}{4} \sin 2 \pi t\left[\begin{array}{c}
a_{21}+3 a_{03} \\
b_{21}+3 b_{03}
\end{array}\right] \\
& +\frac{1}{4} \cos 6 \pi t\left[\begin{array}{c}
a_{30}-a_{12} \\
b_{30}-b_{12}
\end{array}\right]-\frac{1}{4} \sin 6 \pi t\left[\begin{array}{c}
a_{21}-a_{03} \\
b_{21}-b_{03}
\end{array}\right] .
\end{aligned}
$$

We select the Fourier coefficients of this function corresponding to $\cos 2 \pi t$ and $\sin 2 \pi t$ :

$$
y_{c}=\frac{1}{4}\left[\begin{array}{c}
3 a_{30}+a_{12} \\
3 b_{30}+b_{12}
\end{array}\right], y_{s}=-\frac{1}{4}\left[\begin{array}{c}
a_{21}+3 a_{03} \\
b_{21}+3 b_{03}
\end{array}\right] .
$$

Then, according (20), the first number in (55) becomes

$$
\Delta_{0}=\frac{1}{8}\left[3 a_{30}+a_{12}+b_{21}+3 b_{03}\right]=\frac{1}{8}\left[3\left(a_{30}+b_{03}\right)+\left(a_{12}+b_{21}\right)\right] .
$$


5.2.2. Second step. At the second step we let $a_{3}(x) \equiv 0$. Then

$$
f_{3}(t)=T_{0} a_{2 x}^{\prime}(e(t)) e^{T_{0} A t} \int_{0}^{t} e^{-T_{0} A \tau} a_{2}(e(\tau)) d \tau .
$$

Here we have

$$
\begin{aligned}
& e^{T_{0} A t}=\left[\begin{array}{cc}
\cos 2 \pi t & \sin 2 \pi t \\
-\sin 2 \pi t & \cos 2 \pi t
\end{array}\right], \\
& a_{2}(e(\tau))=\frac{1}{2}\left[\begin{array}{c}
a_{20}+a_{02} \\
b_{20}+b_{02}
\end{array}\right]+\frac{1}{2} \cos 4 \pi t\left[\begin{array}{c}
a_{20}-a_{02} \\
b_{20}-b_{02}
\end{array}\right]-\frac{1}{2} \sin 4 \pi t\left[\begin{array}{l}
a_{11} \\
b_{11}
\end{array}\right], \\
& a_{2 x}^{\prime}(e(t))=\cos 2 \pi t\left[\begin{array}{ll}
2 a_{20} & a_{11} \\
2 b_{20} & b_{11}
\end{array}\right]-\sin 2 \pi t\left[\begin{array}{ll}
a_{11} & +2 a_{02} \\
b_{11} & +2 b_{02}
\end{array}\right] .
\end{aligned}
$$

Substituting these expression into (57) and after appropriate calculations, we select the Fourier coefficients of this function corresponding to $\cos 2 \pi t$ and $\sin 2 \pi t$ :

$$
\begin{aligned}
& y_{c}=\frac{1}{12}\left[\begin{array}{c}
10 a_{20} b_{20}+14 a_{20} b_{02}-3 a_{11} a_{20}-3 a_{11} a_{02}+a_{11} b_{11}-4 a_{02} b_{20}+4 a_{02} b_{02} \\
10 b_{20}^{2}+10 b_{20} b_{02}-7 b_{11} a_{20}-5 b_{11} a_{02}+4 b_{20} a_{11}+b_{11}^{2}+2 b_{02} a_{11}+4 b_{02}^{2}
\end{array}\right], \\
& y_{s}=\frac{1}{12}\left[\begin{array}{c}
-5 b_{20} a_{11}-7 b_{02} a_{11}+10 a_{02} a_{20}+10 a_{02}^{2}+4 a_{20}^{2}+2 b_{11} a_{20}+a_{11}^{2}+4 b_{11} a_{02} \\
-3 b_{11} b_{20}-3 b_{11} b_{02}+14 a_{20} b_{02}+10 a_{02} b_{02}+4 a_{20} b_{20}-4 a_{02} b_{20}+a_{11} b_{11}
\end{array}\right] .
\end{aligned}
$$

Then, according (20), the first number in (55) becomes

$$
\begin{aligned}
\Delta_{0} & =\frac{1}{24}\left\{6 a_{20} b_{20}+3 b_{11} b_{20}+3 b_{11} b_{02}-3 a_{11} a_{20}-3 a_{11} a_{02}-6 a_{02} b_{02}\right\} \\
& =-\frac{1}{8}\left\{\left[\left(a_{11} a_{02}+2 a_{02} b_{02}\right)-\left(2 a_{20} b_{20}+b_{11} b_{20}\right)-\left(b_{11} b_{02}-a_{11} a_{20}\right)\right] .\right.
\end{aligned}
$$

In the general case when the right hand side of system $(52)$ involves both vector functions $a_{2}(x)$ and $a_{3}(x)$, the number $\Delta_{0}$ is the sum of numbers (56) and 58

5.2.3. Comparison of $\Delta_{0}$ with Lyapunov value $L_{1}$. We consider equation (52), in which the matrix $A$ is of form

$$
A=\left[\begin{array}{cc}
a & b \\
c & -a
\end{array}\right]
$$

and $\omega^{2}=-a^{2}-b c>0$. In [1], the first Lyapunov value for this equation was provided in the form:

$$
\begin{aligned}
L_{1}= & -\frac{1}{8 b \omega^{2}}\left\{\left[a c\left(a_{11}^{2}+a_{11} b_{02}+a_{02} b_{11}\right)+a b\left(b_{11}^{2}+a_{20} b_{11}+a_{11} b_{20}\right)\right.\right. \\
& +c^{2}\left(a_{11} a_{02}+2 a_{02} b_{02}\right)-2 a c\left(b_{02}^{2}-a_{20} a_{02}\right)-2 a b\left(a_{20}^{2}-b_{20} b_{02}\right) \\
& \left.-b^{2}\left(2 a_{20} b_{20}+b_{11} b_{20}\right)+\left(b c-2 a^{2}\right)\left(b_{11} b_{02}-a_{11} a_{20}\right)\right] \\
& \left.-\left(a^{2}+b c\right)\left[3\left(c b_{03}-b a_{30}\right)+2 a\left(a_{21}+b_{12}\right)+\left(c a_{12}-b b_{21}\right)\right]\right\} .
\end{aligned}
$$

We note that the cited work has a misprint in the formula for $L_{1}$ : it should be divided by the number $2 \pi / \omega$.

Substituting $a=0, b=-c=1$, we obtain

$$
L_{1}=-\frac{1}{8}\left\{\left[\left(a_{11} a_{02}+2 a_{02} b_{02}\right)-\left(2 a_{20} b_{20}+b_{11} b_{20}\right)-\left(b_{11} b_{02}-a_{11} a_{20}\right)\right]+\left[3\left(-b_{03}-a_{30}\right)+\left(-a_{12}-b_{21}\right)\right]\right\} \text {. }
$$

Comparing the numbers $\Delta_{0}$ and $L_{1}$, we see that these numbers coincide for system (52).

To complete the proof of Theorem 5 , it remains to show that the numbers $(22)$ are independent of the choice of the vectors $e, g, e^{*}, g^{*}$ in accordance with identities (14). Assume that 
we are given another set of the vectors $e, g, e^{*}, g^{*}$. We can show that each another set of the vectors can be described as

$e_{1}=e \cos \varphi+g \sin \varphi, \quad g_{1}=g \cos \varphi-e \sin \varphi, \quad e_{1}^{*}=e^{*} \cos \varphi+g^{*} \sin \varphi, \quad g_{1}^{*}=g^{*} \cos \varphi-e^{*} \sin \varphi$

for some $\varphi$. Substituting these vectors into (22), it is easy to confirm that numbers (22) have the same values for each $\varphi$. The proof of Theorem 5 is complete.

5.3. Proof of Theorem 10. We shall need an auxiliary statement, which can be proved by straightforward calculations and which is of an independent interest. We let

$$
B_{1}=I-A_{0}, \quad B_{2}=I+A_{0}+P_{0} .
$$

By construction, the operators $B_{1}: \mathbb{R}^{N} \rightarrow \mathbb{R}^{N}$ and $B_{2}: \mathbb{R}^{N} \rightarrow \mathbb{R}^{N}$ are invertible and the subspaces $E_{0}$ and $E^{0}$ are invariant for them.

Lemma 2. Assume that the matrix $A_{0}$ possesses the simple eigenvalue -1 and the absolute values of its other eigenvalues are less or greater than 1 . Then the central manifold $W_{c}$ of system (36) can be described by the identity

$$
W_{c}=\{x: x=\varepsilon e+\psi(\varepsilon)\},
$$

where

$$
\psi(\varepsilon)=\varepsilon^{2} \psi_{2}+\varepsilon^{3} \psi_{3}+\widehat{\psi}_{4}(\varepsilon) .
$$

Here the coefficients $\psi_{2}$ and $\psi_{3}$ are determined by the identities

$$
\psi_{2}=B_{1}^{-1} P^{0} a_{2}, \quad \psi_{3}=B_{2}^{-1} P^{0}\left[-2\left(a_{2}, g\right)\left(A_{0} \psi_{2}+a_{2}\right)-a_{2}^{\prime} \psi_{2}-a_{3}\right],
$$

and the function $\widehat{\psi}_{4}(\varepsilon)$ is smooth and satisfies the relation: $\left\|\widehat{\psi}_{4}(\varepsilon)\right\|=O\left(\varepsilon^{4}\right), \varepsilon \rightarrow 0$.

For the sake of simplicity we restrict ourselves by considering the situation when system (36) is two-dimensional, that is, $N=2$. For the sake of simplicity we also assume that as $\mu=\mu_{0}$, the matrix $A(\mu)$ is of the form

$$
A_{0}=A\left(\mu_{0}\right)=\left[\begin{array}{cc}
-1 & 0 \\
0 & b
\end{array}\right]
$$

where $b \neq \pm 1$. Finally, assume that as $\mu=\mu_{0}$ the quadratic and cubic terms in nonlinearity (33) are respectively of form:

$$
\begin{aligned}
& a_{2}(x)=\left[\begin{array}{c}
a_{20} x_{1}^{2}+2 a_{11} x_{1} x_{2}+a_{02} x_{2}^{2} \\
b_{20} x_{1}^{2}+2 b_{11} x_{1} x_{2}+b_{02} x_{2}^{2}
\end{array}\right], \\
& a_{3}(x)=\left[\begin{array}{c}
a_{30} x_{1}^{3}+3 a_{21} x_{1}^{2} x_{2}+3 a_{12} x_{1} x_{2}^{2}+a_{03} x_{2}^{3} \\
b_{30} x_{1}^{3}+3 b_{21} x_{1}^{2} x_{2}+3 b_{12} x_{1} x_{2}^{2}+b_{03} x_{2}^{3}
\end{array}\right] .
\end{aligned}
$$

We have

$$
\begin{array}{lll}
e=g=\left[\begin{array}{l}
1 \\
0
\end{array}\right], & P_{0}=\left[\begin{array}{ll}
1 & 0 \\
0 & 0
\end{array}\right], & P^{0}=\left[\begin{array}{ll}
0 & 0 \\
0 & 1
\end{array}\right], \\
a_{2}=\left[\begin{array}{l}
a_{20} \\
b_{20}
\end{array}\right], & a_{2}^{\prime}=2\left[\begin{array}{ll}
a_{20} & a_{11} \\
b_{20} & b_{11}
\end{array}\right], & a_{3}=\left[\begin{array}{l}
a_{30} \\
b_{30}
\end{array}\right] .
\end{array}
$$

First we determine the form of equation (35) as $\mu=\mu_{0}$ in the considered case. In order to do this, we first observe that system (34) for system (36) is of form:

$$
\left\{\begin{array}{l}
u_{n+1}=P_{0}\left[A(\mu)\left(u_{n}+v_{n}\right)+a\left(u_{n}+v_{n}, \mu\right)\right], \\
v_{n+1}=P^{0}\left[A(\mu)\left(u_{n}+v_{n}\right)+a\left(u_{n}+v_{n}, \mu\right)\right],
\end{array}\right.
$$


where $u_{n}=P_{0} x_{n}$ and $v_{n}=P^{0} x_{n}$; both equations in this system are scalar. Letting $u_{n}=\varepsilon_{n} e$, we obtain that as $\mu=\mu_{0}$, equation (35) is equivalent to the scalar equation

$$
\varepsilon_{n+1}=-\varepsilon_{n}+\left(a\left(\varepsilon_{n} e+\psi\left(\varepsilon_{n}\right), \mu_{0}\right), g\right) .
$$

Thus, as $\mu=\mu_{0}$, the right hand side of equation (35) is of form:

$$
G\left(\varepsilon, \mu_{0}\right)=-\varepsilon+\left(a\left(\varepsilon e+\psi(\varepsilon), \mu_{0}\right), g\right) .
$$

Due to identities (33) and (60), we can show easily that for small $\varepsilon$ we have

$$
G\left(\varepsilon, \mu_{0}\right)=-\varepsilon+\varepsilon^{2}\left(a_{2}, g\right)+\varepsilon^{3}\left[\left(a_{2}^{\prime} \psi_{2}, g\right)+\left(a_{3}, g\right)\right]+O\left(\varepsilon^{4}\right) .
$$

It was noted in [1] that if as $\mu=\mu_{0}$, here the right hand side of equation (35) is of form

$$
G\left(\varepsilon, \mu_{0}\right)=-\varepsilon+\gamma_{2} \varepsilon^{2}+\gamma_{3} \varepsilon^{3}+O\left(\varepsilon^{4}\right),
$$

then the first Lyapunov value is determined by the formula:

$$
l_{1}=-\left(\gamma_{2}^{2}+\gamma_{3}\right)
$$

coinciding with formula (39); in fact, there is a misprint in the formula of $l_{1}$ in the cited work, the sign should be opposite.

It remains to confirm that numbers (37) and (64) (where $\gamma_{2}=\left(a_{2}, g\right)$ and $\gamma_{3}=\left[\left(a_{2}^{\prime} \psi_{2}, g\right)+\right.$ $\left.\left.\left(a_{3}, g\right)\right]\right)$ coincide. Taking into consideration formulae (61), by straightforward calculations we obtain that 37) and (64) are equal to the same number:

$$
l_{1}=-\left(a_{20}^{2}+a_{30}+\frac{2}{1-b} a_{11} b_{20}\right) .
$$

The proof is complete.

5.4. Proof of Theorem 12, We restrict ourselves by proving the first formula in (44). Assume that as $\mu=\mu_{0}$, the function $a_{3}(x, \mu)$ in nonlinearity (43) is determined by identity (63). For this case, in [2], there was given the following formula for Lyapunov value $L_{1}$ :

$$
L_{1}=\frac{3}{8}\left[\left(a_{30}+a_{12}+b_{21}+b_{03}\right) \cos 2 \pi \theta_{0}+\left(b_{30}+b_{12}-a_{21}-a_{03}\right) \sin 2 \pi \theta_{0}\right] .
$$

This is why the proof of the first formula in (44) reduces to substituting (63) into (44) and calculating the corresponding integral. As a result, we obtain the number coinciding with (65).

\section{BIBLIOGRAPHY}

1. L.P. Shil'nikov, A.L. Shil'nikov, D.V. Turaev, L. Chua. Methods of qualitative theory in nonlinear dynamics. II. Institut Kompyuter. Issledov., Moscow-Izhevsk (2009). [World Scientific Series on Nonlinear Science. Series A 5. World Scientific Publishing, Singapore (2001).]

2. J. Guckenheimer, P.J. Holmes. Nonlinear oscillations, dynamical systems, and bifurcations of vector fields. Springer, New York (1983).

3. V.A. Pliss. Integral sets of periodic systems of differential equations. Nauka, Moscow (1977). (in Russian).

4. A. Kelley. The stable, center-stable, center-unstable, unstable manifolds // J. Diff. Eq. 3:4, 546570 (1967).

5. A.D. Bruno. Analytic form of differential equations. I, II // Trudy Mosk. Matem. Obsch. 25, 119-162 (1971); 26, 199-239 (1972). (in Russian).

6. Sh.-N. Chow, Ch. Li, D. Wang. Normal forms and bifurcation of planar vector fields. Cambridge Univ. Press, Cambirdge (1994).

7. Yu.A. Kuznetsov. Elements of applied bifurcation theory. Springer, New York (1998).

8. G.A. Leonov, N.V. Kuznetsov, E.V. Kudryashova. A direct method for calculating Lyapunov values of two-dimensional dynamical systems // Trudy IMM UrO RAN. 16:1, 119-126 (2010). [Proc. Steklov Inst. Math. Suppl. 1. 272, S119-S126 (2011).] 
9. S. Lynch. Symbolic computation of Lyapunov quantities and the second part of Hilbert's sixteenth problem // in "Differential equations with symbolic computations", eds. D. Wang, Zh. Zheng. Birkhäuser, Basel (2005).

10. A.A. Vyshinskiy, L.S. Ibragimova, S.A. Murtazina, M.G. Yumagulov. An operator method for approximately studying regular bifurcation in multiparameter dynamical systems // Ufimskij Matem. Zhurn. 2:4, 3-26 (2010).

11. L.S. Ibragimova L.S. I.G. Fanina, M.G. Yumagulov. The asymptotic formulae for the problem of the construction hyperbolic and stability regions of dynamical systems // Ufimskij Matem. Zhurn. 8:3, 59-81 (2016). [Ufa Math. J. 8:3, 58-78 (2016).]

12. M.G. Yumagulov. Localization of Arnold tongues of discrete dynamical systems // Ufimskij Matem. Zhurn. 5:2, 109-131 (2013). [Ufa Math. J. 5:2, 109-131 (2013).]

13. M.G. Yumagulov. An operator method for studying regular bifurcations in multiparameter systems // Dokl. Akad. Nauk. 424:2, 177-180 (2009). [Dokl. Math. 79:1, 41-44 (2009).]

14. M.A. Krasnosel'skij, M.G. Yumagulov. The parameter functionalization method in the eigenvalue problem // Dokl. Akad. Nauk. 365:2, 162-164 (1999). [Dokl. Math. 59:2, 204-206 (1999).]

15. J.E. Marsden, M. McCracken. The Hopf bifurcation and its applications. Applied Math. Sci. 19. Springer-Verlag, New York (1976).

16. N.N. Bautin. Behavior of dynamical systems near the boundaries of stability domains. Gostekhizdat, Leningrad (1949). (in Russian).

17. M.A. Krasnoselski. Shift operator along trajectories of differential equations. Nauka, Moscow (1966). (in Russian).

Nadezhda Ivanovna Gusarova,

Rybinsk State Aviation Technical University

named after P.A. Soloviev,

Pushkin str., 53,

152934, Rybinsk, Jaroslav region, Russia

E-mail: gusarova-73@mail.ru

Sariya Ashirafovna Murtazina,

Sibai institut (branch) of Bashkir State University,

Belova str., 21,

453833, Sibai, Russia

E-mail: sariamurtaz@mail.ru

Marat Flyurovich Fazlytdinov,

Bashkir State University,

Zaki Validi str., 32,

450076, Ufa, Russia

E-mail: fazlitdin_marat@mail.ru

Marat Gayazovich Yumagulov,

Bashkir State University,

Zaki Validi str., 32,

450076, Ufa, Russia

E-mail: yum_mg@mail.ru 